\title{
Evaluation and Compensation of Detector Solenoid Effects on Disrupted Beam in the ILC 14 mrad Extraction Line*
}

\author{
Dragan Toprek \\ VINCA Institute of Nuclear Sciences, 11001 Belgrade, Serbia \\ Yuri Nosochkov \\ SLAC National Accelerator Laboratory, 2575 Sand Hill Road, Menlo Park, CA 94025, USA
}

\begin{abstract}
This paper presents calculations of detector solenoid effects on disrupted primary beam in the ILC 14 mrad extraction line. Particle tracking simulations are performed for evaluation of primary beam loss along the line as well as of beam distribution and polarization at Compton Interaction Point. The calculations are done both without and with solenoid compensation. The results are obtained for the baseline ILC energy of $500 \mathrm{GeV}$ center-of-mass and three options of beam parameters.
\end{abstract}

* Work supported by the U.S. Department of Energy contract DE-AC02-76SF00515. 


\title{
Evaluation and Compensation of Detector Solenoid Effects on Disrupted Beam in the ILC 14 mrad Extraction Line*
}

\author{
Dragan Toprek \\ VINCA Institute of Nuclear Sciences, 11001 Belgrade, Serbia \\ Yuri Nosochkov \\ SLAC National Accelerator Laboratory, Menlo Park, CA 94025, USA
}

\begin{abstract}
This paper presents calculations of detector solenoid effects on disrupted primary beam in the ILC 14 mrad extraction line. Particle tracking simulations are performed for evaluation of primary beam loss along the line as well as of beam distribution and polarization at Compton Interaction Point. The calculations are done both without and with solenoid compensation. The results are obtained for the baseline ILC energy of $500 \mathrm{GeV}$ center-of-mass and three options of beam parameters.
\end{abstract}

\section{INTRODUCTION}

The ILC baseline extraction line is designed for 14 mrad horizontal crossing angle between $\mathrm{e}^{+}$and $\mathrm{e}-$ colliding beams at Interaction Point (IP) [1]. The extraction optics provides large beam acceptance in order to minimize beam losses caused by large angular spread and long energy tail in the disrupted primary and secondary beams. It also includes dedicated vertical chicanes for beam energy measurement and gamma calorimeter (GAMCAL) diagnostics, and a low $\beta$ focal point with $2 \mathrm{~cm}$ vertical dispersion used as a Compton Interaction Point (CIP) for polarization measurement.

The crossing angle scheme naturally results in a horizontal angle between beam trajectory and direction of the detector solenoid field $B_{s}$, equal to half-crossing angle $\theta_{c}=7 \mathrm{mrad}$. This angle creates a systematic horizontal field component $B_{x}=B_{s} \sin \theta_{c}$ on the beam trajectory which causes vertical beam deflection and therefore produces vertical orbit and dispersion, synchrotron radiation and rotation of beam polarization vector. In addition, the solenoid field creates a weak focusing effect in $x-y$ planes and coupling of $x-y$ betatron motion which alter the downstream beam properties.

Perturbation of the extracted beam caused by the solenoid needs to be evaluated and compensated in order to avoid a higher beam loss and preserve the desired beam properties at the Compton IP for polarization measurement. This paper presents calculations of solenoid optics effects using MAD code [2] and tracking simulations of primary electron beam using DIMAD code [3]. The results are focused on evaluation of solenoid effects on disrupted beam loss in magnets and collimators, and beam distribution and polarization at Compton IP. The calculations are done for the push-pull version of extraction optics [4,5] with the final focus drift of $L^{*}=3.51 \mathrm{~m}$ and with the $\mathrm{SiD}$ [6,7] detector solenoid field model, for the nominal ILC energy of $500 \mathrm{GeV}$ center-of-mass (CM) and three options of beam parameters.

\section{EXTRACTION LINE OPTICS}

This section briefly reviews the optics of the $14 \mathrm{mrad}$ extraction line [4]. The length of the extraction line from IP to dump is about $300 \mathrm{~m}$. Horizontal view of the incoming and extraction magnets on one side of IP is shown in Fig. 1. The large 14 mrad crossing angle allows the extracted beam to continue straight ahead without the need for bends in the

\footnotetext{
* Work supported by the U.S. Department of Energy contract DE-AC02-76SF00515.
} 
horizontal plane. In this configuration, the first superconducting (SC) incoming quadrupole QD0 is located at distance of $L^{*}=3.51 \mathrm{~m}$ from IP, and the first extraction SC quadrupole QDEX1 is at $5.5 \mathrm{~m}$ to provide sufficient horizontal separation from the incoming line. These two quadrupoles and the incoming sextupole SD0 will be part of a detector in a push-pull configuration [5]. The long warm section after these magnets provides the necessary space for detector exchange in push-pull operation. Beam apertures of the extraction SC quadrupoles QDEX1 and QFEX2A are set to the maximum values of $R=15 \mathrm{~mm}$ and $30 \mathrm{~mm}$, respectively, limited by the separation from the incoming magnets. A long drift after the QFEX2A provides the necessary transverse space for the incoming crab cavity. The remaining downstream extraction quadrupoles are warm magnets starting at distance of $17.19 \mathrm{~m}$ from IP. The extraction quadrupole system is designed to provide: 1 ) a low $\beta$ focal point at $148.6 \mathrm{~m}$ from IP, where the Compton IP will be located, 2) the optimal transformation term $R_{22}=-0.5$ from IP to CIP for efficient polarization measurement, and 3) large chromatic and geometric acceptance for keeping the disrupted beam loss at acceptable level.

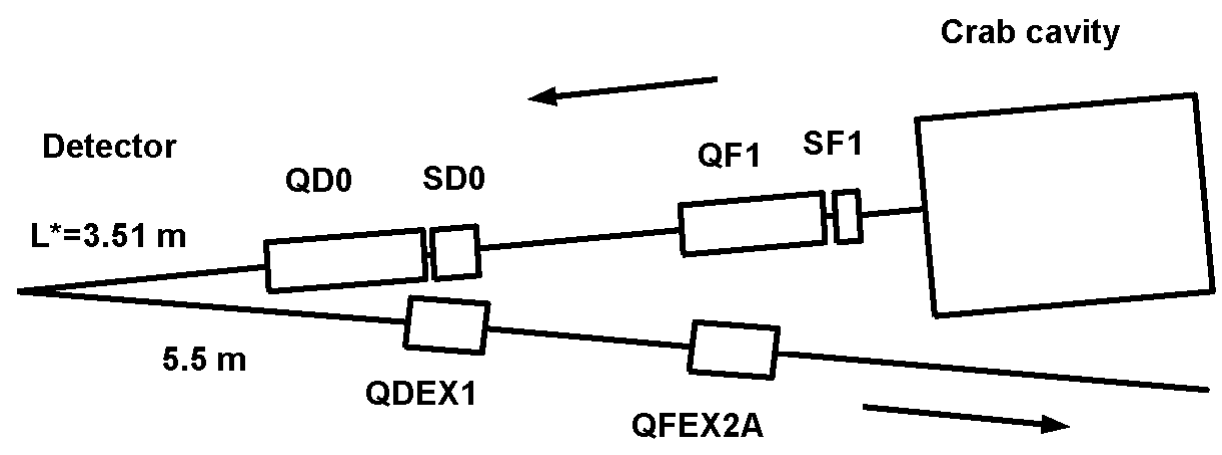

Figure 1: Horizontal view of incoming and extraction magnets on one side of IP.

Downstream of the quadrupoles, the extraction line includes a four bend vertical chicane for measurements of beam energy (Fig. 2), a four bend vertical chicane for polarization measurement, and two vertical bends for gamma calorimeter luminosity diagnostics (Fig. 3) [8]. The polarimeter bends create $2 \mathrm{~cm}$ vertical dispersion at the Compton IP located at center of the polarimeter chicane. After the last GAMCAL bend magnet, the extraction line contains a set of 5 horizontal and 5 vertical fast kickers, located on average $\sim 85 \mathrm{~m}$ before the dump. The rapidly oscillating kicker field ( $\sim 1 \mathrm{kHz}$ ) will sweep the beam along a $R=3 \mathrm{~cm}$ circle at the dump in order to increase the effective beam area for protecting the dump window from high power density of small undisrupted beam and preventing water boiling in the dump vessel.

The extraction collimation system includes two collimators in the chicanes. The first one is placed at center of the energy chicane, $60.7 \mathrm{~m}$ after IP, where momentum dispersion is $17 \mathrm{~mm}$. Its $40 \mathrm{~mm}$ vertical aperture on the low energy side of the beam is set to remove the low energy tail electrons with relative energies below $30-35 \%$. The second collimator at $160.9 \mathrm{~m}$ is inside the polarimeter chicane to protect the Cherenkov detector (at $175.6 \mathrm{~m}$ ) from synchrotron radiation created in the energy chicane bends. Three more collimators (COLW1, COLW2 and COLW3) are included in the final $100 \mathrm{~m}$ section before the dump in order to protect the fast sweeping kickers and limit beam size to within $R=$ $15 \mathrm{~cm}$ at the dump window. 


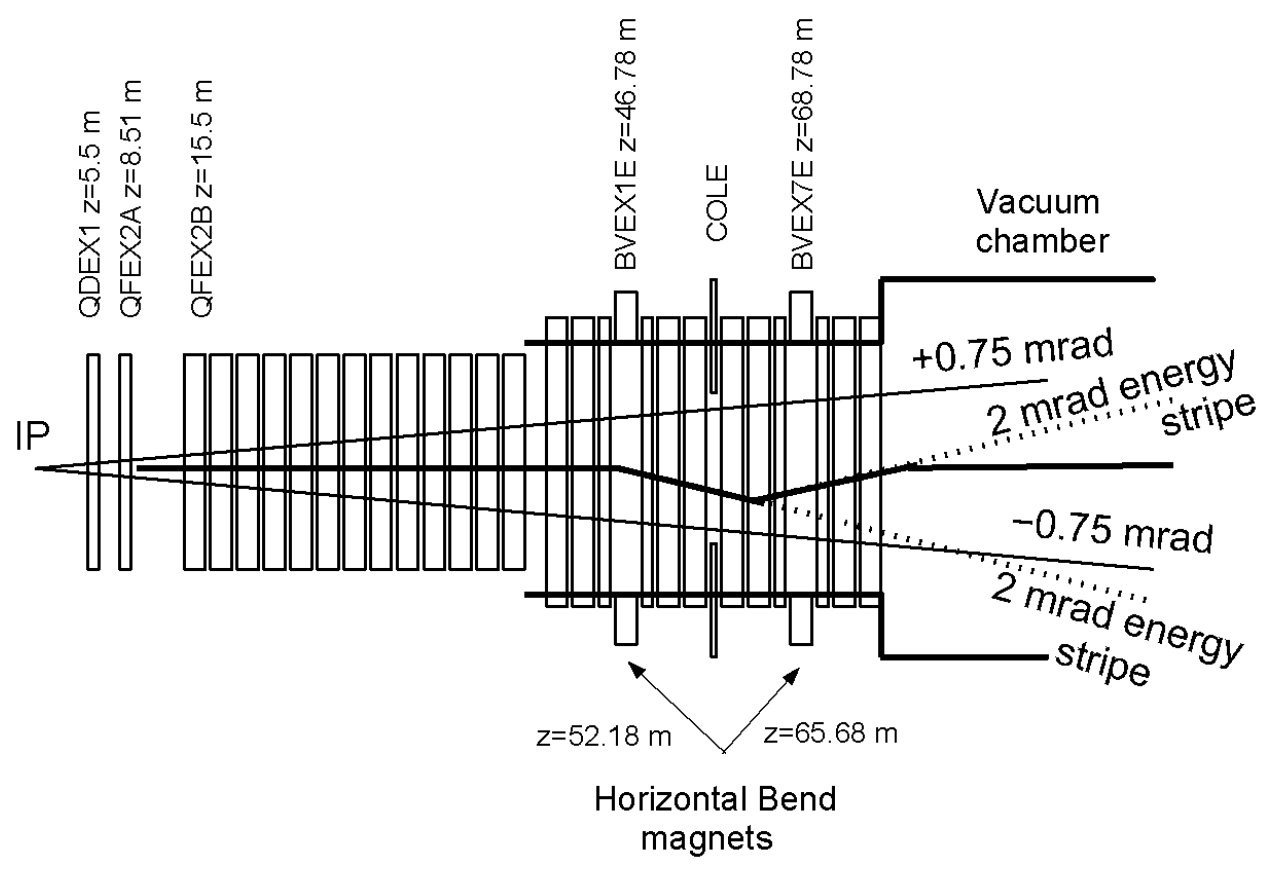

Figure 2: Vertical view of the extraction quadrupoles and energy chicane bends, where z-distances are from IP. $\pm \mathbf{0 . 7 5}$ mrad beam stay clear for beamstrahlung photons is also shown [8].

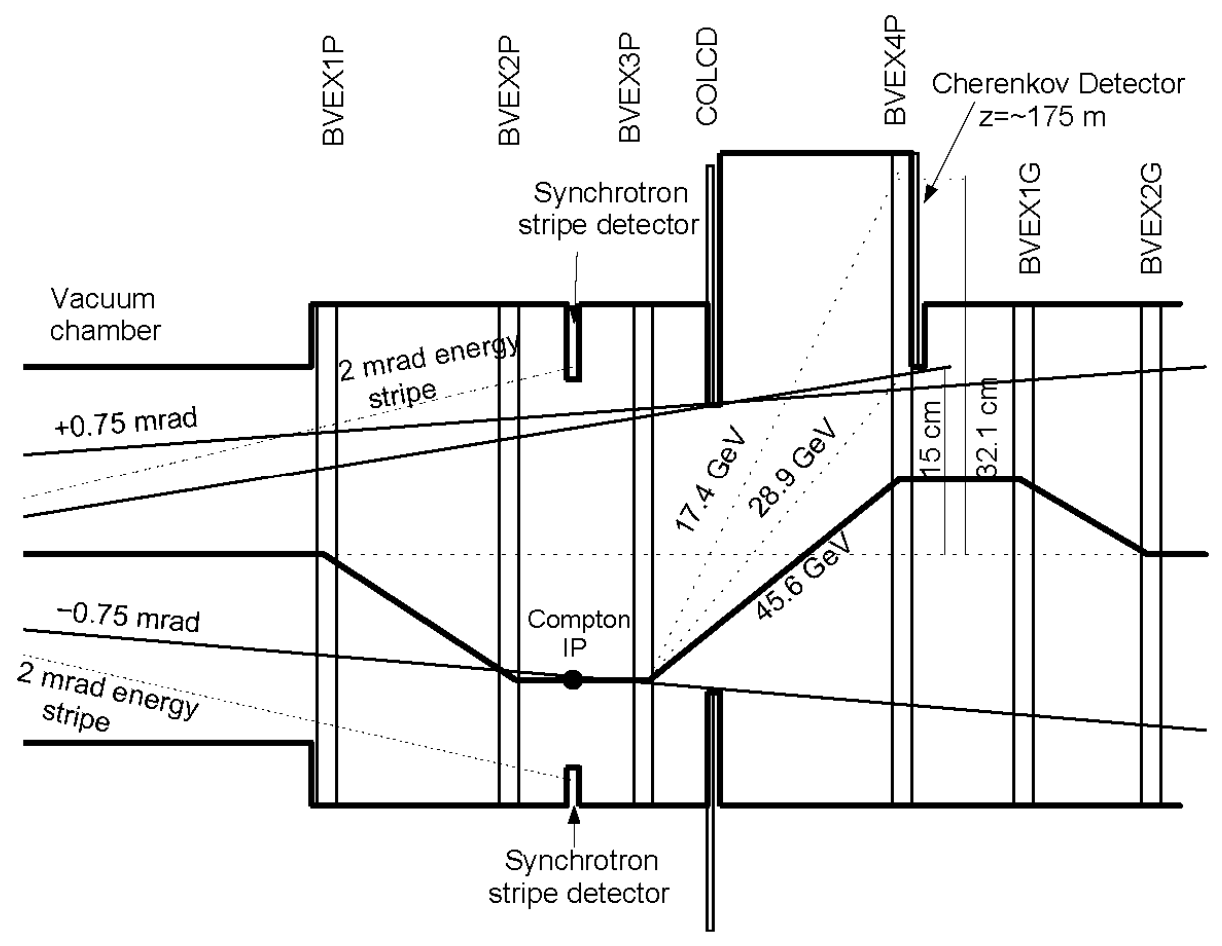

Figure 3: Vertical view of the four polarimeter bends and two GAMCAL bends [8]. \pm 0.75 mrad beam stay clear for beamstrahlung photons is also shown. 


\section{DISRUPTED BEAM PROPERTIES AT IP}

\subsection{Parameter Options}

Distributions of primary disrupted beams at IP were generated using GUINEA-PIG beam-beam simulation code [9]. Beam disruption in collision creates a very long tail of low energy particles and large angular divergence in the outgoing beams. Three beam parameter options were studied [10,11]: 1) nominal (C11), 2) with a large vertical emittance (C13), and 3) with a low beam power (C14). These parameter settings are based on different values of emittance, beam current and beam size at IP, but they keep the same design luminosity. Consequently, the effect of beam disruption varies in these options. The low power option C14 yields the strongest disruption effect leading to a larger extracted beam. The disruption can be further enhanced by a vertical offset between the e+ and e- beams at IP. Therefore, for each parameter option two cases were studied: without a vertical offset and with a vertical offset corresponding to maximum disruption effect. The selected full beam-to-beam vertical offsets $\Delta y$ in the C11, C13, C14 options are $200 \mathrm{~nm}, 300 \mathrm{~nm}$, and $120 \mathrm{~nm}$, respectively. Note that in the machine operation any IP offsets will be actively compensated to minimize their effects on luminosity and beam disruption. A horizontal beam offset is not studied in this paper since it reduces the disruption effect for flat beams [12] and therefore is not a concern for extracted beam. Beam parameters used in this study are listed in Table 1 [10].

Table 1: Beam parameters at IP for $500 \mathrm{GeV}$ CM energy.

\begin{tabular}{|l|c|c|c|}
\hline Parameter & $\begin{array}{c}\text { Nominal } \\
\text { C11 }\end{array}$ & $\begin{array}{c}\text { Large Y-Emit } \\
\text { C13 }\end{array}$ & $\begin{array}{c}\text { Low Power } \\
\text { C14 }\end{array}$ \\
\hline Number of particles per bunch, $N$ & $2 \cdot 10^{10}$ & $2 \cdot 10^{10}$ & $2 \cdot 10^{10}$ \\
\hline Number of bunches per train, $N_{b}$ & 2820 & 2820 & 1330 \\
\hline Repetition frequency, $f[\mathrm{~Hz}]$ & 5 & 5 & 5 \\
\hline Norm. emittance, $\gamma \varepsilon_{\mathrm{x}}[\mathrm{m} \cdot \mathrm{rad}]$ & $1 \cdot 10^{-5}$ & $1.2 \cdot 10^{-5}$ & $1 \cdot 10^{-5}$ \\
\hline Norm. emittance, $\gamma \varepsilon_{\mathrm{y}}[\mathrm{m} \cdot \mathrm{rad}]$ & $4 \cdot 10^{-8}$ & $8 \cdot 10^{-8}$ & $3.5 \cdot 10^{-8}$ \\
\hline Beta function, $\beta_{\mathrm{x}}{ }^{*}[\mathrm{~mm}]$ & 21 & 10 & 10 \\
\hline Beta function, $\beta_{\mathrm{y}}{ }^{*}[\mathrm{~mm}]$ & 0.4 & 0.4 & 0.2 \\
\hline RMS beam size, $\sigma_{\mathrm{x}}{ }^{*}[\mathrm{~nm}]$ & 655 & 495 & 452 \\
\hline RMS beam size, $\sigma_{\mathrm{y}}{ }^{*}[\mathrm{~nm}]$ & 5.7 & 8.1 & 3.8 \\
\hline RMS bunch length, $\sigma_{\mathrm{z}}{ }^{*}[\mu \mathrm{m}]$ & 300 & 500 & 200 \\
\hline Energy loss by beamstrahlung, $\delta_{\mathrm{BS}}$ & 0.022 & 0.024 & 0.057 \\
\hline Beam power, $P[\mathrm{MW}]$ & 11.3 & 11.3 & 5.3 \\
\hline Luminosity, $\mathcal{L}\left[\mathrm{cm}^{-2} \mathrm{~s}^{-1}\right]$ & $2 \cdot 10^{34}$ & $2 \cdot 10^{34}$ & $2 \cdot 10^{34}$ \\
\hline
\end{tabular}

Tracking of $\sim 10^{5}$ particles is typically sufficient for adequate simulation of beam distribution in the extraction line and for calculation of relatively high beam loss in collimators. However, accurate estimate of much lower losses in 
magnets typically requires a much higher statistics of $>10^{7}$ particles in the simulated beam. The latter, unfortunately, significantly increases the simulation running time and requires large computer storage space. Fortunately, for the purpose of beam loss calculation in magnets, it is not necessary to track a full beam because only very low energy particles are lost in extraction magnets due to over-focusing and dispersion. In this case, it is sufficient to track only the lowest part of beam energy tail and particles with large initial $x-y$ angles. As a result, two types of particle data files were used in this study. The low statistics files containing total of $\sim 710^{4}$ particles in two beams were used for evaluation of beam distribution and polarization at Compton IP, and for calculation of high beam loss in extraction collimators. The high statistics files with total of up to $3.5 \cdot 10^{7}$ particles in two beams were generated for the purpose of beam loss calculation in extraction magnets. But for tracking simulations, these files were reduced to much smaller files containing $\leq 10^{5}$ particles with relative energies below 65\% and/or initial $x-y$ angles larger than \pm 0.5 mrad. For maximum statistics, the above files contain both $\mathrm{e}+$ and $\mathrm{e}-$ colliding beam distributions, but assuming the same charge for all particles. In case of ideal collisions without beam offset at IP, the $\mathrm{e}^{+}$and $\mathrm{e}-$ disrupted distributions are identical, therefore combining the two beams does not make difference. In case of a non-zero IP vertical offset, the outgoing $\mathrm{e}^{+}$ and e- beams will have the same but opposite systematic offsets of $y$ and $y^{\prime}$ at IP. In this case, combining e+ and ebeams is equivalent to combining two beams with initial positive and negative vertical offsets. Therefore, the resultant power loss will be an average of power losses with positive and negative offsets at IP.

The disrupted beam distributions at IP for the Nominal (C11), Large Vertical Emittance (C13), and Low Power (C14) options without and with vertical offset are shown in Fig. 4-9, obtained by GUINEA-PIG. The use of combined beam files is apparent in Fig. 5,7,9 with non-zero IP $y$-offset, where the two beams are separated in $y$ and $y^{\prime}$ directions. Distributions of particle energies are presented in Fig. 10, where logarithmic scale helps to see the long low energy tail.
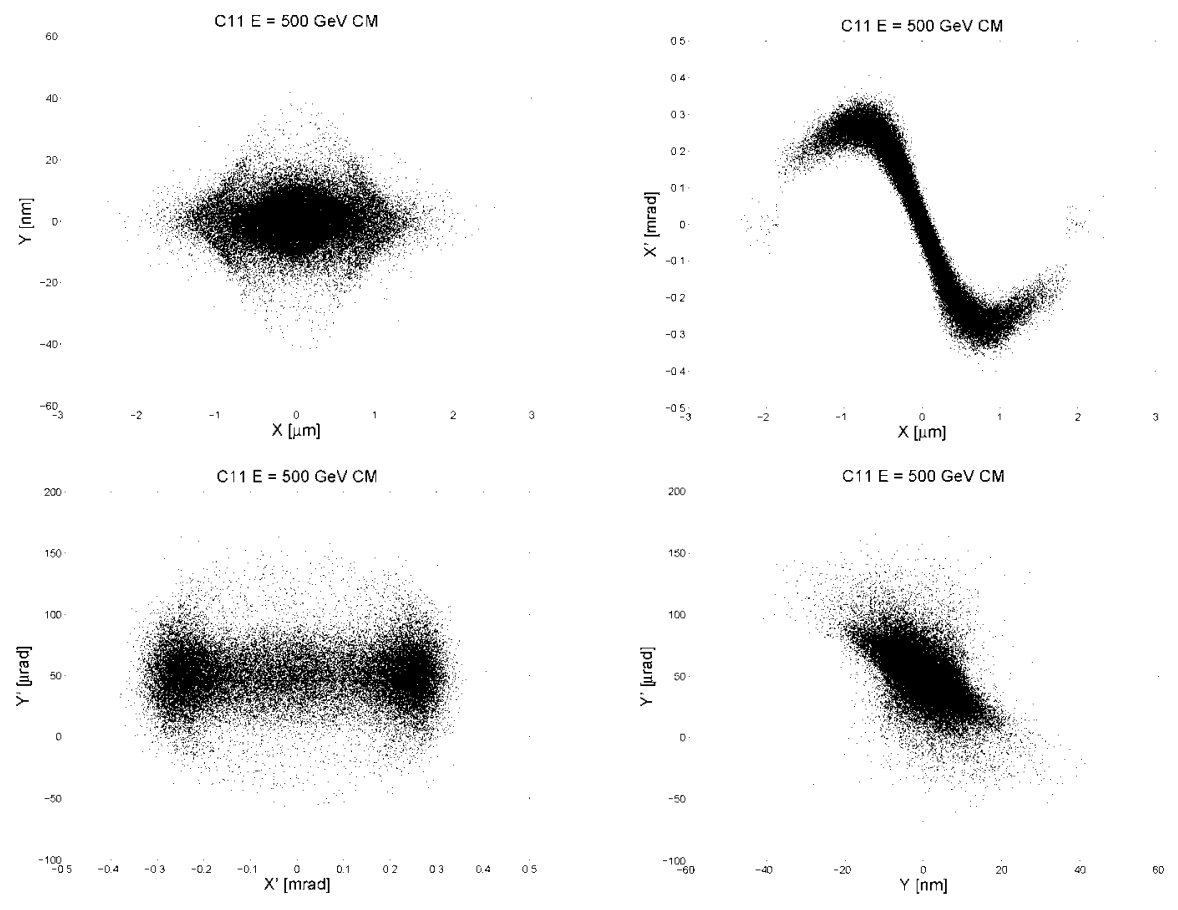

Figure 4: Disrupted beam distribution at IP for the Nominal parameter option (C11). 

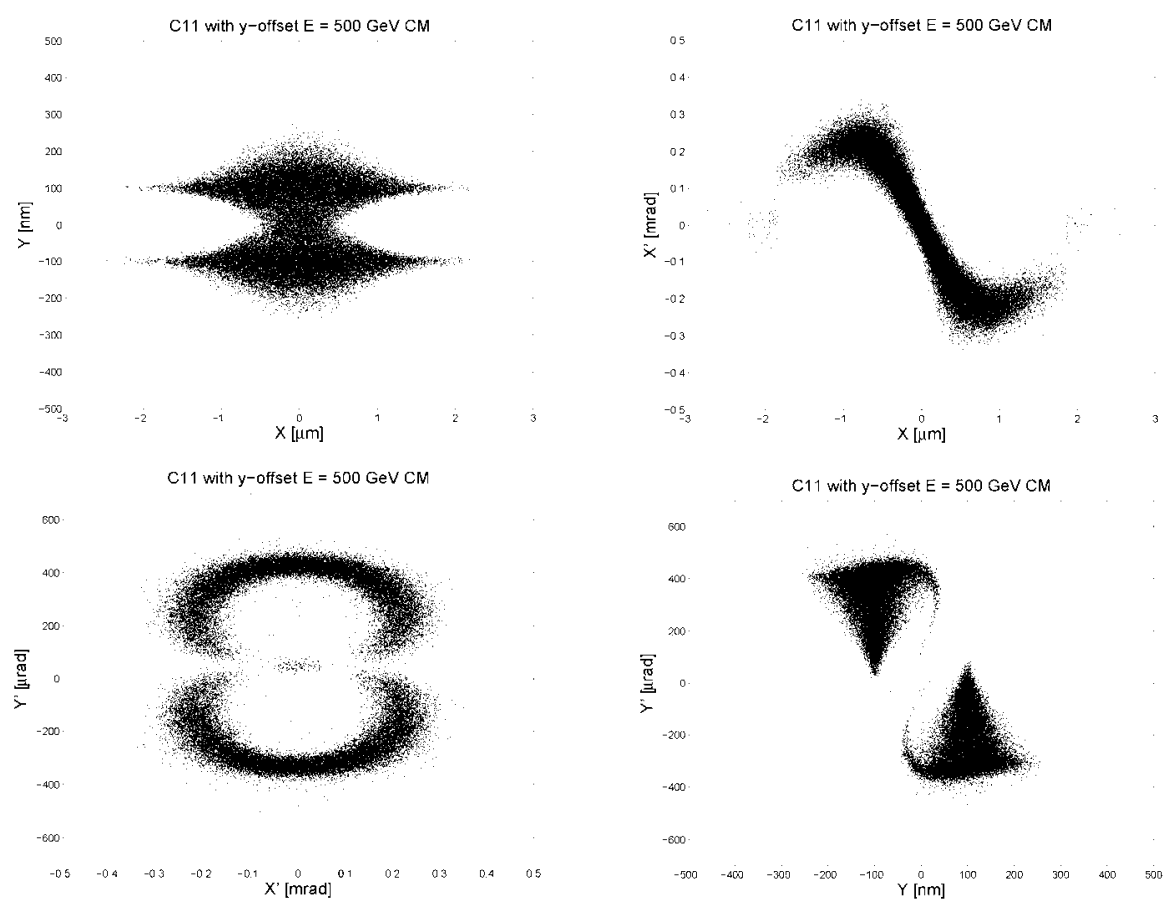

Figure 5: Disrupted beam distribution at IP for the Nominal parameter option (C11) with $200 \mathrm{~nm} y$-offset.
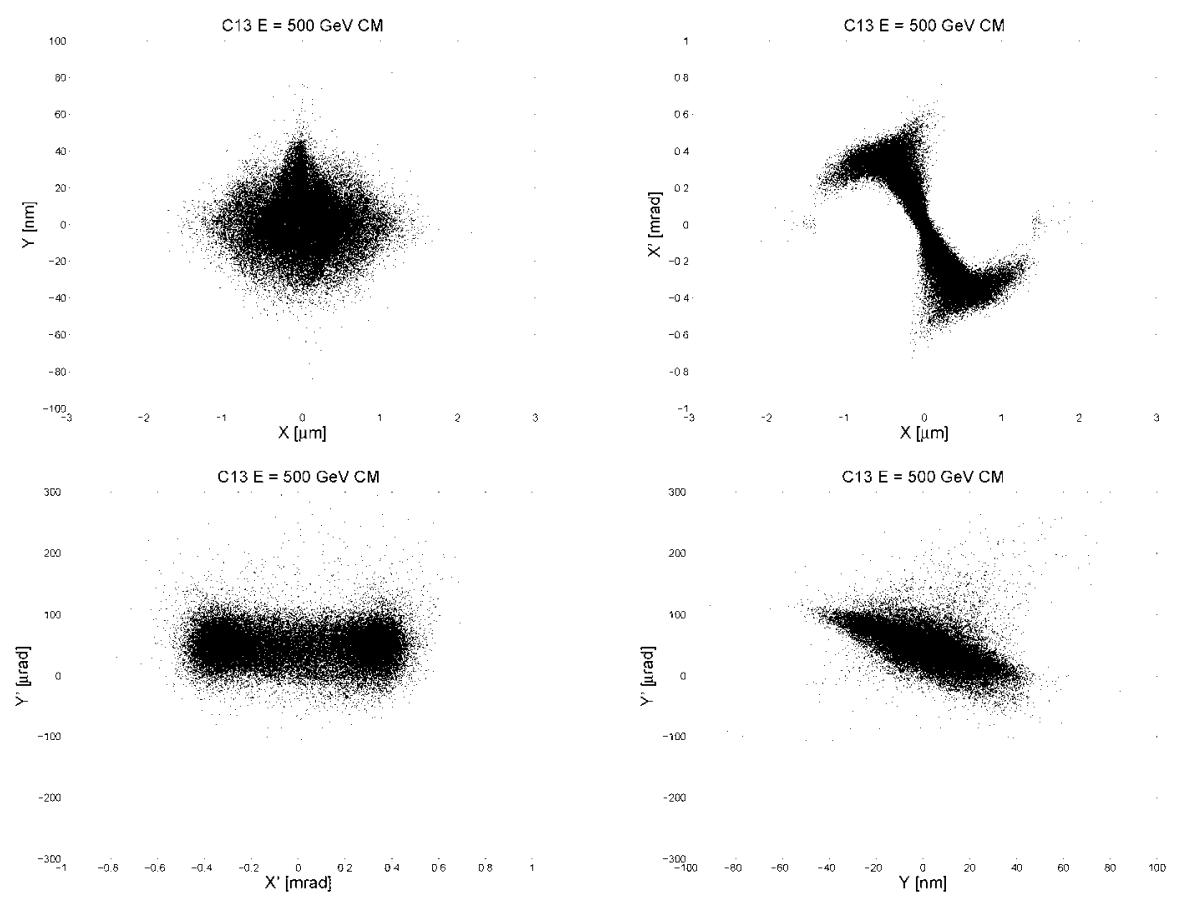

Figure 6: Disrupted beam distribution at IP for the Large Y-emittance parameter option (C13). 

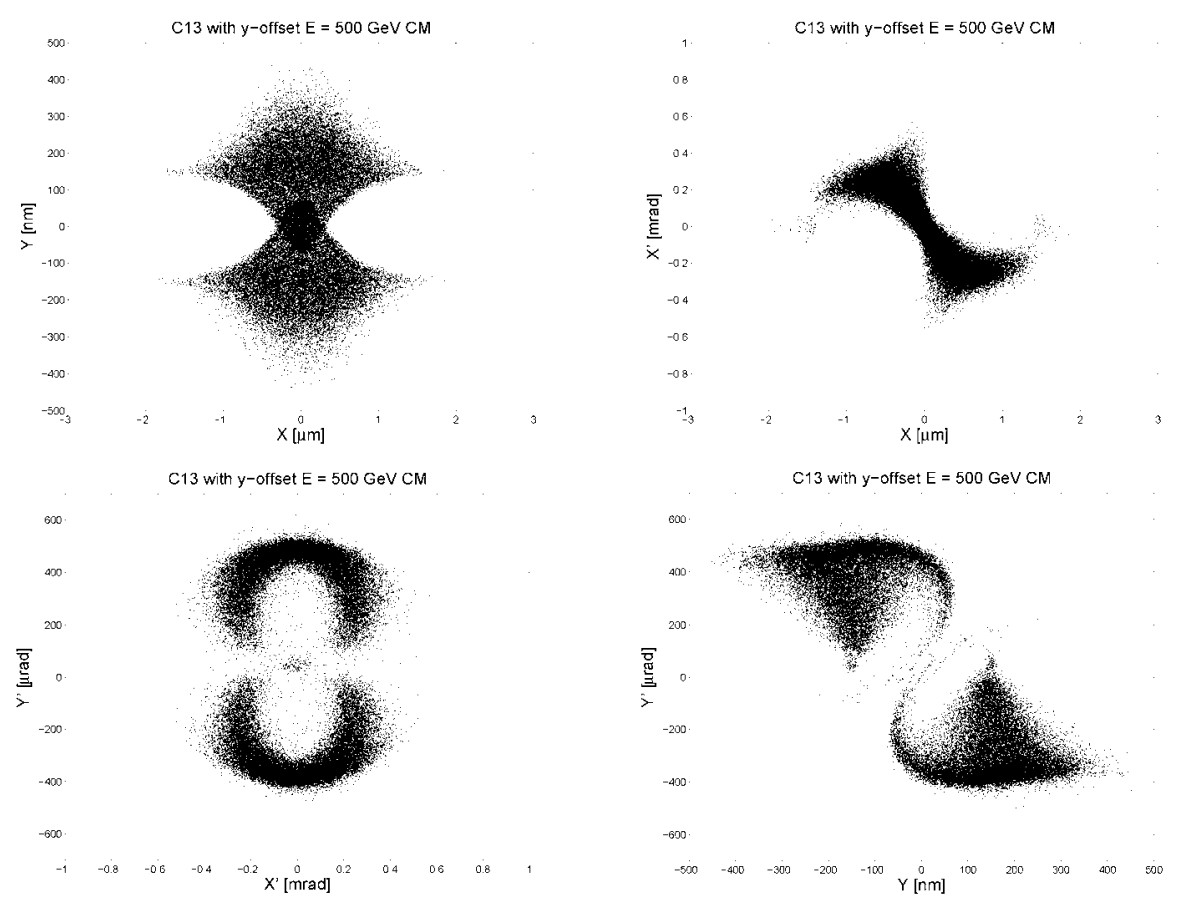

Figure 7: Disrupted beam distribution at IP for the Large Y-emittance parameter option (C13) with $300 \mathrm{~nm} y$ offset.
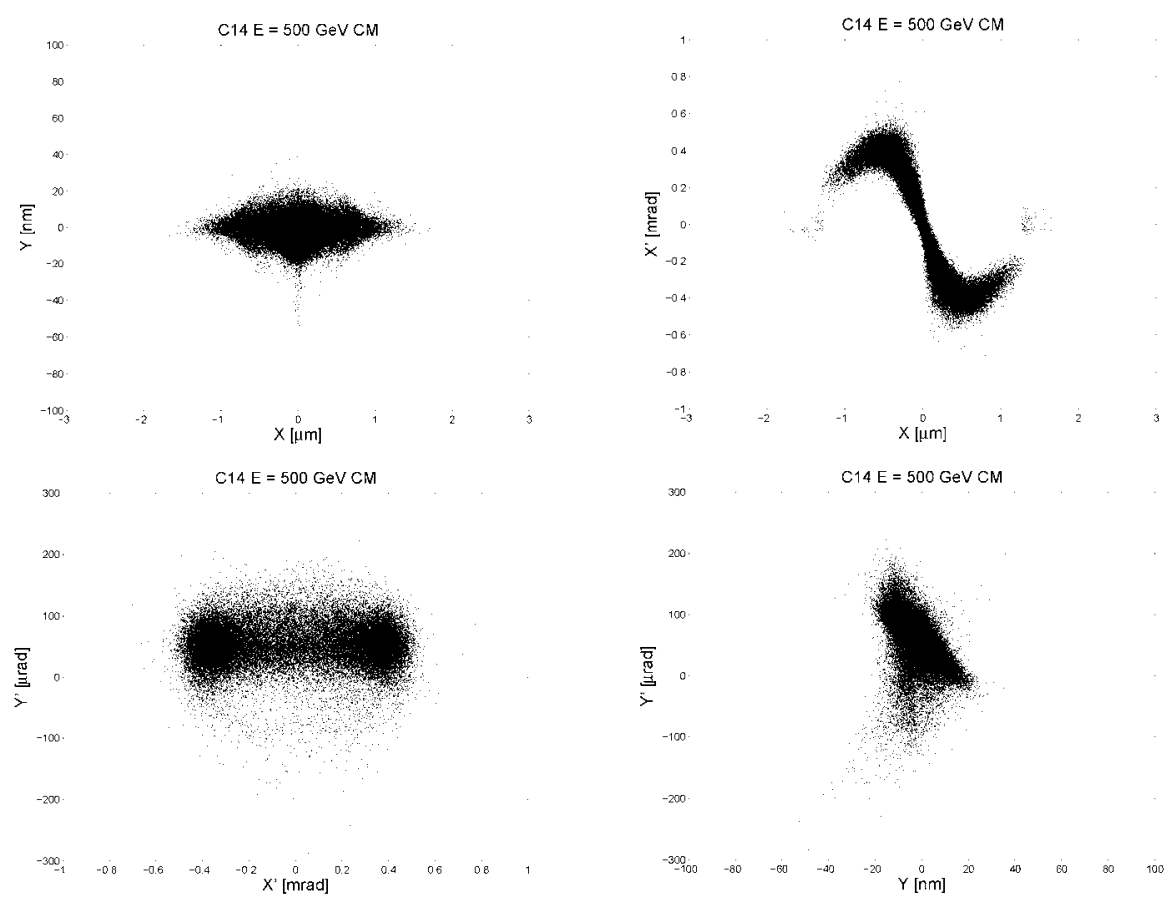

Figure 8: Disrupted beam distribution at IP for the Low Power parameter option (C14). 

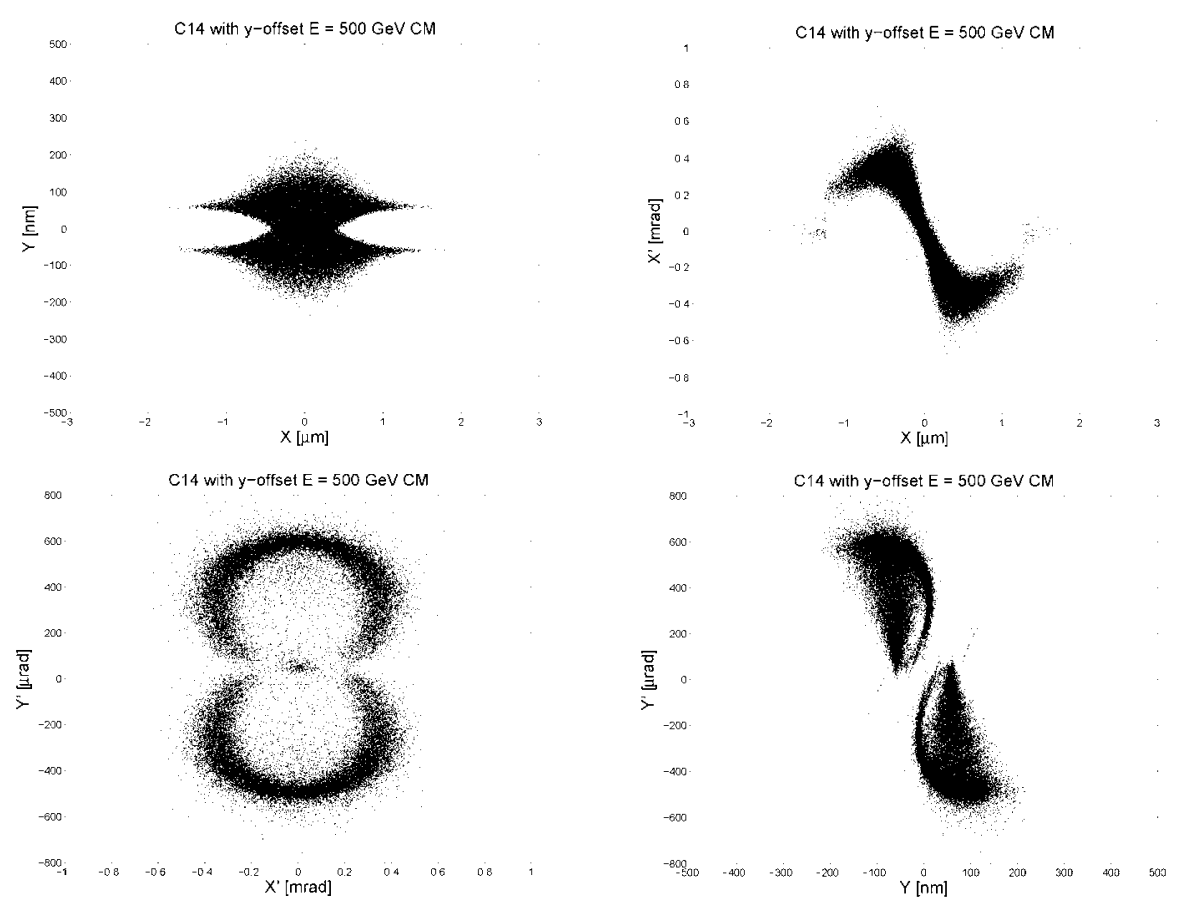

Figure 9: Disrupted beam distribution at IP for the Low Power parameter option (C14) with $120 \mathrm{~nm}$ y-offset.

\subsection{Luminosity Weighted Polarization at IP}

The luminosity weighted polarization at IP is approximated by [13,14]:

$$
P_{\text {Luminosity Weighted }}=\cos \left(\theta_{\text {SpinLuminosityWeighted }}\right)=\cos \left(\frac{E[G e V]}{0.44065} \cdot \frac{1}{2} \theta_{\text {angle }}(I P)\right)
$$

where $\theta_{\text {angle }}(I P)$ is angle of a particle as it leaves the IP:

$$
\theta_{\text {angle }}(I P)=\sqrt{x^{\prime 2}+y^{\prime 2}}
$$

Based on the GUINEA-PIG simulated disrupted beam, the distributions of luminosity weighted polarization at IP were calculated for the Nominal, Large Vertical Emittance and Low Power parameter options without (left) and with (right) vertical offset and are presented in Fig.11.

\section{EFFECTS OF DETECTOR SOLENOID FIELD WITHOUT COMPENSATION}

The non-zero horizontal crossing angle at IP results in both the longitudinal and horizontal field components $\left(B_{s}\right.$ and $B_{x}=B_{s} \sin \theta_{c}$ ) produced by detector solenoid field on beam trajectory. The $B_{s}$ field creates a weak focusing and $x-y$ coupling of betatron motion; and the $B_{x}$ component generates a systematic vertical beam deflection which produces vertical orbit, synchrotron radiation and rotation of beam polarization vector. Perturbations caused by detector solenoid need to be evaluated and compensated in order to avoid a potential increase in beam loss and preserve the desired beam properties at Compton IP for polarization measurement. 

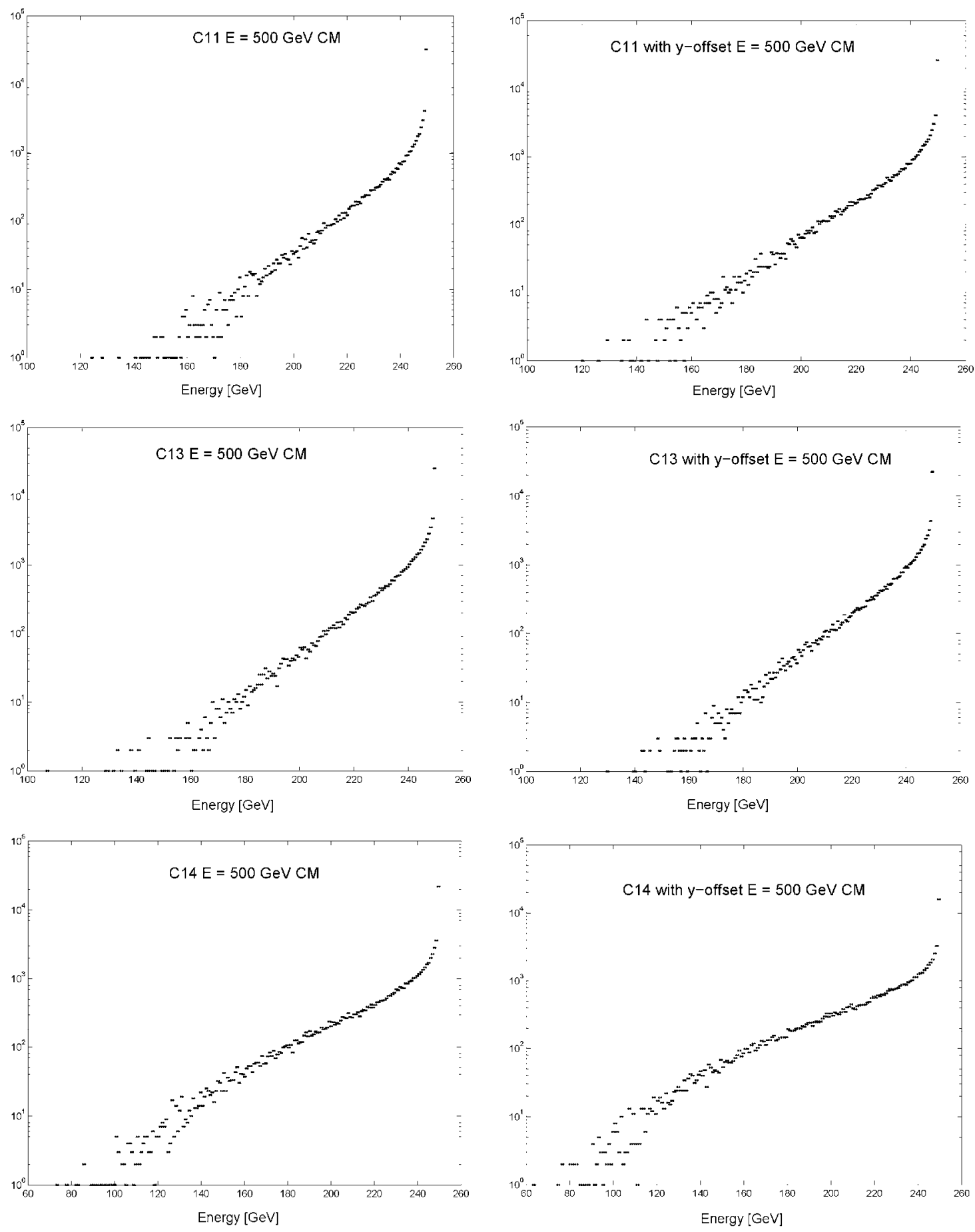

Figure 10: Disrupted energy distributions at IP for the Nominal (top), Large Y-emittance (middle) and Low Power (bottom) parameter options without (left) and with (right) IP $y$-offset. 
C11, E $=500 \mathrm{GeV} \mathrm{CM}$
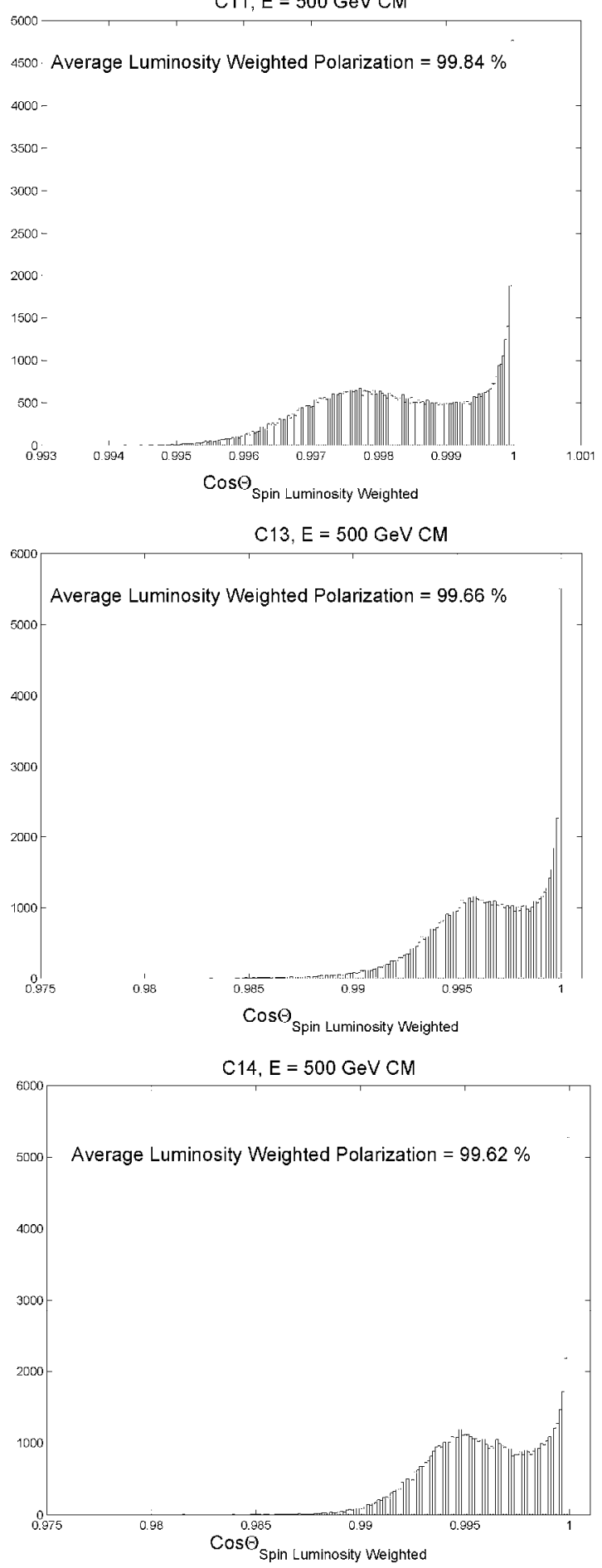

C11 with y-offset, $E=500 \mathrm{GeV} C M$

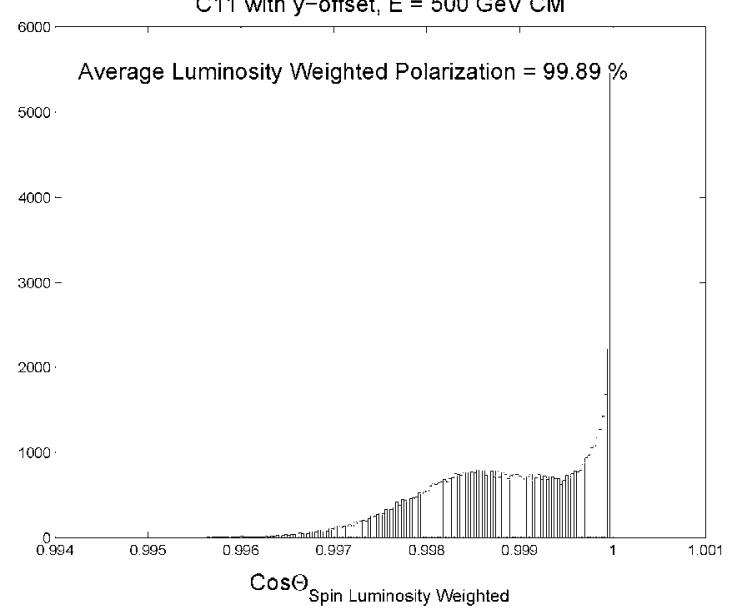

C13 with $y$-offset $E=500 \mathrm{GeV}$ CM

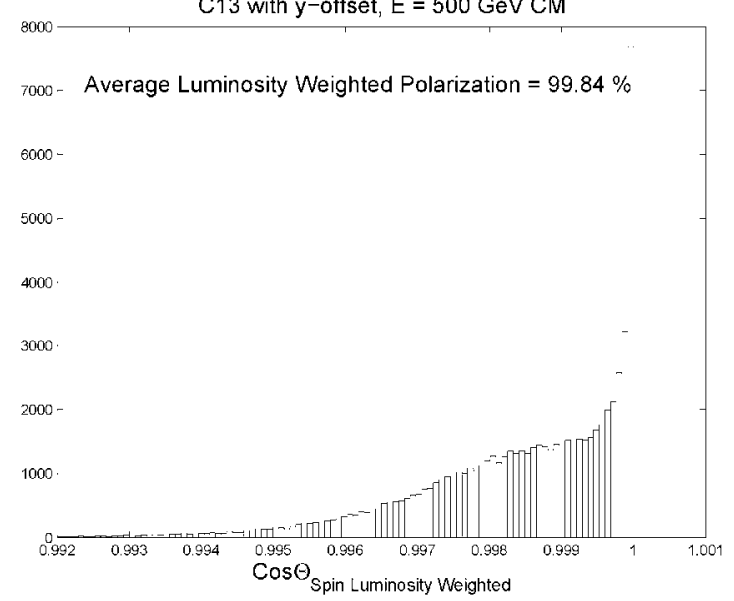

C14 with $y$-offset, $E=500 \mathrm{GeV} \mathrm{CM}$

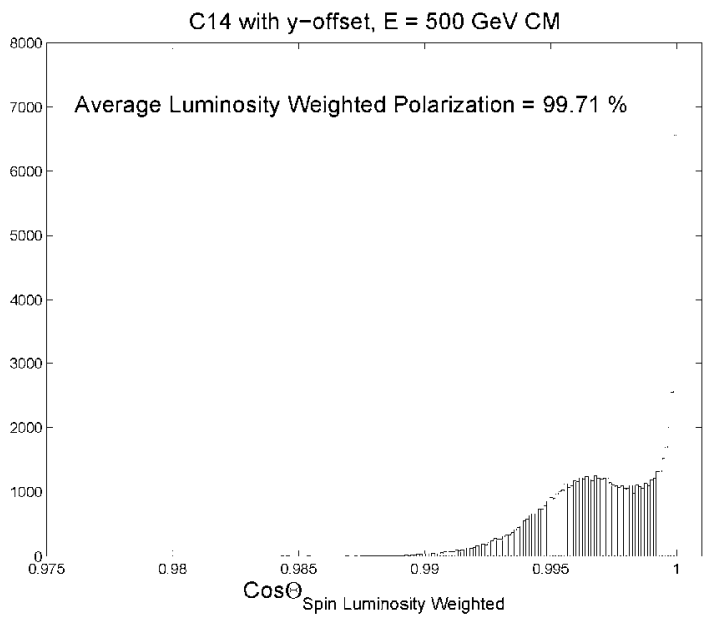

Figure 11: Luminosity weighted polarization at IP for the Nominal (top), Large Y-emittance (middle) and Low Power (bottom) parameter options without (left) and with (right) IP $y$-offset. 
In this section, the extraction optics and beam properties at Compton IP are first investigated without a solenoid field, and then with solenoid field without compensation. The solenoid field model in this study is based on the Silicon Detector (SiD) design [6,7].

\subsection{Detector Solenoid Field is OFF}

Fig. 12 shows the disrupted $\beta$ functions and dispersion in the 14 mrad extraction line, as calculated by MAD, for the Nominal parameter option C11, when detector solenoid field is turned off. In this case, the initial orbit at IP is set to zero, and therefore it is zero everywhere in the extraction line since there are no solenoid and other field errors. The initial dispersion is also set to zero since the beam disruption in collision should generally destroy the incoming dispersion. In this case, dispersion remains zero everywhere after IP except in the energy and polarization chicanes where local bumps of vertical dispersion are formed. Beta functions are focused by the extraction quadrupoles to a low $\beta$ waist at the Compton IP ( $s=148.6 \mathrm{~m}$ ) with the transformation matrix term $R_{22}=-0.5$ from IP to CIP, suitable for polarization measurements.

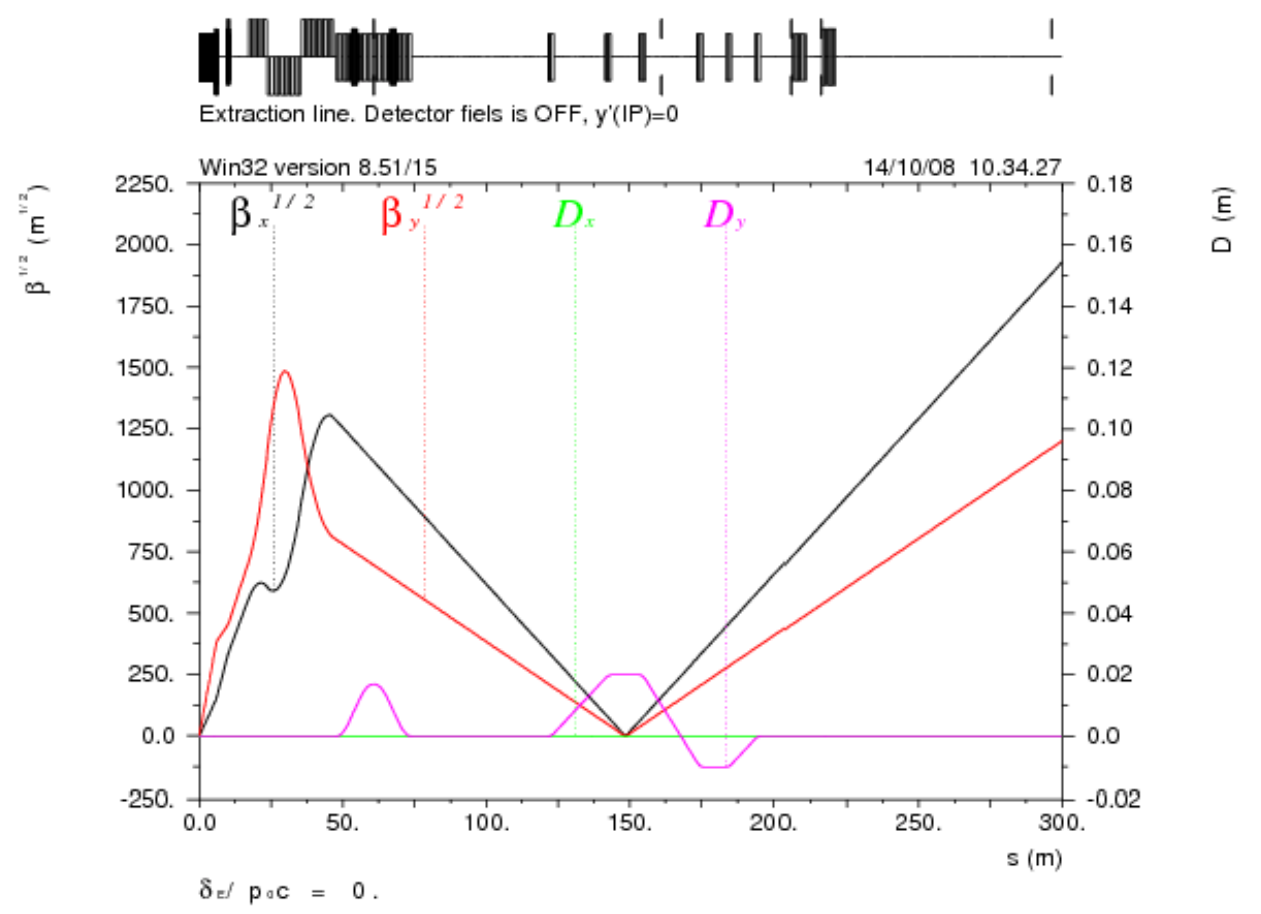

Figure 12: Disrupted $\beta$ functions and dispersion in the 14 mrad extraction line for the Nominal parameter option when solenoid field is turned off.

DIMAD tracking of $\sim 7 \cdot 10^{4}$ primary particles from IP to Compton IP was performed, and the resultant $x-y$ distributions at CIP are shown in Fig. 13 for the Nominal, Large Y-emittance and Low Power parameter options without and with IP vertical offset. The distributions without IP offset are shaped towards the negative $y$-values due to the negative vertical offsets of low energy particles caused by the $2 \mathrm{~cm}$ vertical dispersion at CIP. The negative vertical shift is also present but not clearly seen in the distributions with non-zero IP offset because it is overshadowed by larger 

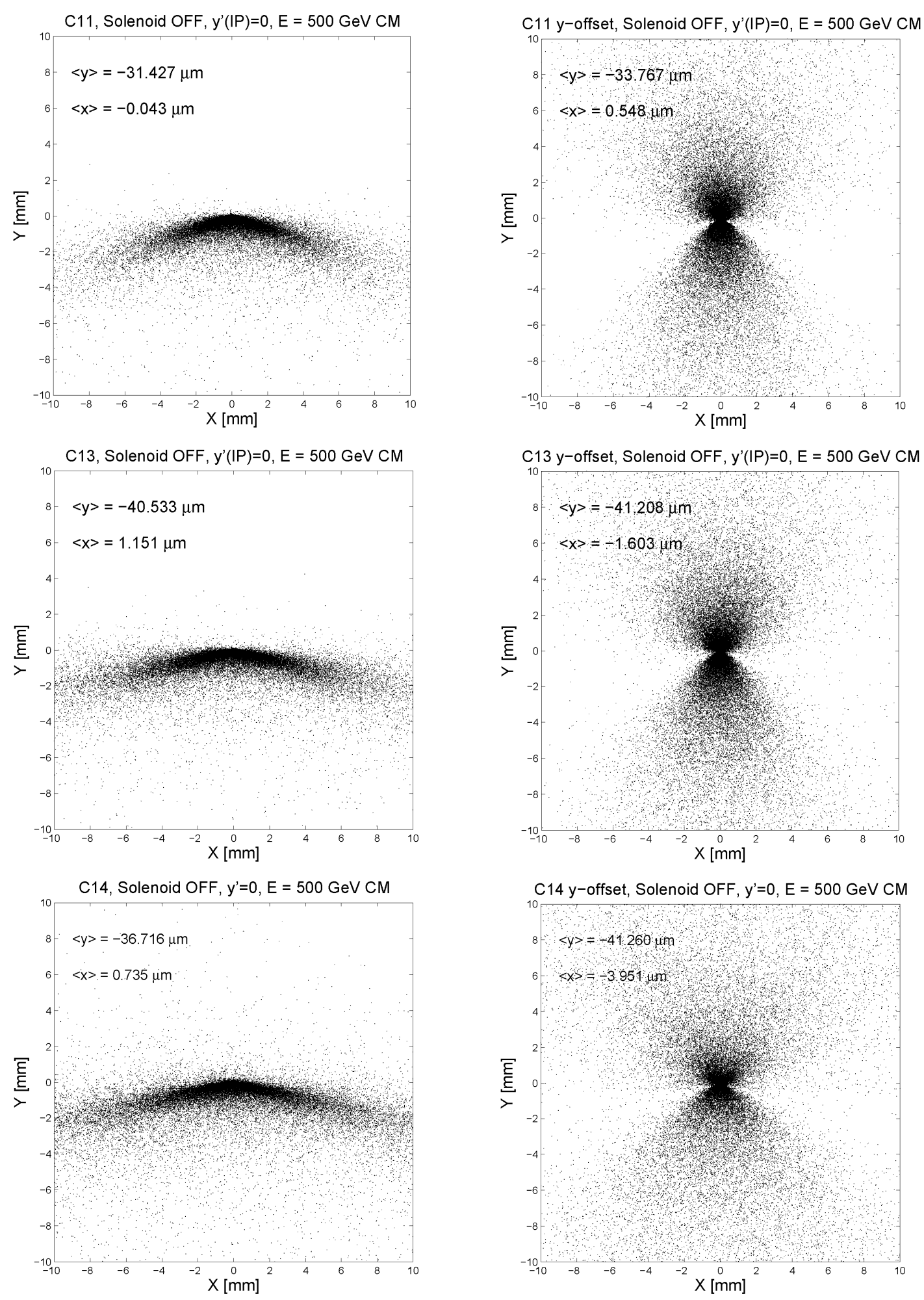

Figure 13: $X-Y$ disrupted beam distributions at Compton IP for the Nominal (top), Large Y-emittance (middle) and Low Power (bottom) parameter options without (left) and with (right) IP $y$-offset, when solenoid field is turned off. Here $<x>$ and $<y>$ are the horizontal and vertical offsets of beam core with $|\Delta p / p|<1 \%$. 
vertical offsets caused by the initial IP $y$-offset. In the latter case, the combined tracked beams which are vertically separated at IP continue to be separated at Compton IP as shown in Fig. 13.

\subsection{Detector Solenoid Field is ON}

The solenoid field model in this study is based on the Silicon Detector (SiD) design [6,7]. The longitudinal and radial solenoid field components on a beam trajectory are shown in Fig. 14 as a function of distance from IP. In our MAD model, the continuous profile of the longitudinal solenoid field is replaced with a set of short solenoid slices, where the field is changed from slice to slice according to the profile in Fig. 14. The radial field components are automatically calculated by MAD once the solenoid slices are properly aligned with respect to beam trajectory.
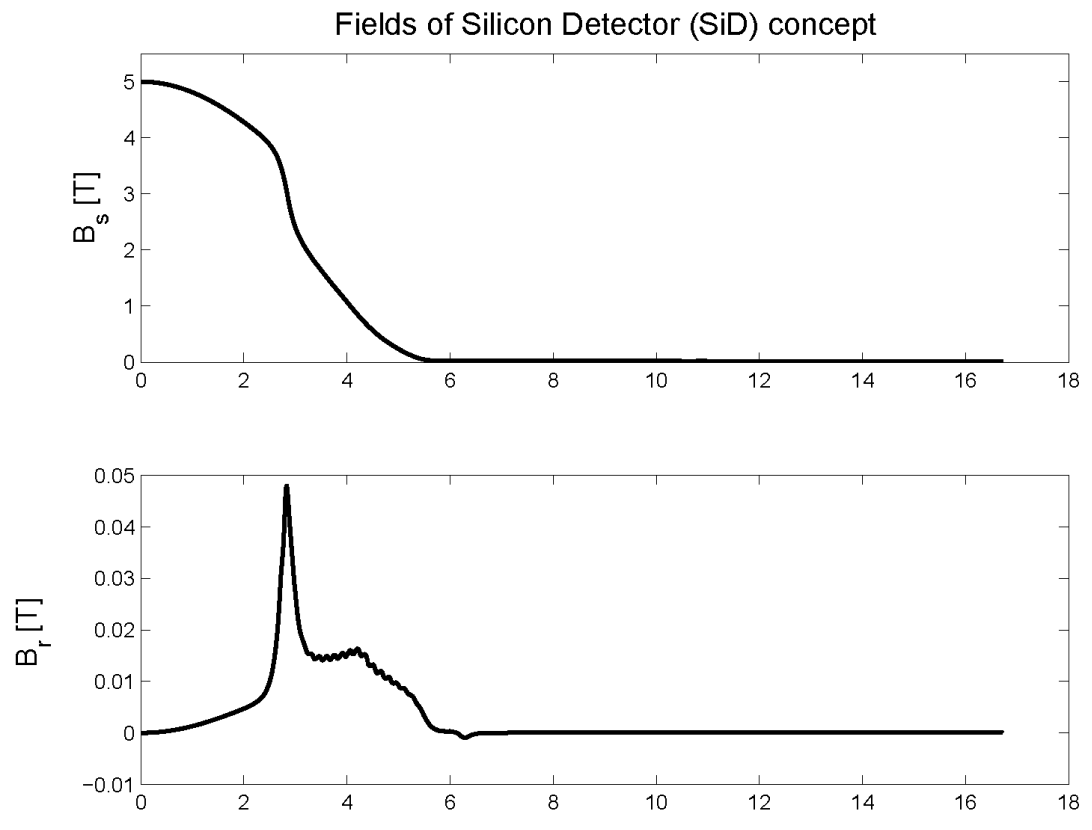

Figure 14: Longitudinal $B_{s}$ and radial $B_{r}$ components of the SiD solenoid field on beam trajectory as a function of distance from IP (in meters).

In this section, the SiD solenoid field is turned on, but its optical effects on extraction beam are not compensated. Additionally, it is assumed that the disrupted beam has a non-zero vertical orbit angle $y^{\prime}(I P)$ at IP. This angle is the result of optimization of incoming beam orbit and SR induced by the solenoid upstream of IP in order to minimize the colliding beam size. Since the exact value of this angle is not decided yet, in this study we use a "reasonable guess" value of $y^{\prime}(I P)=50 \mu \mathrm{rad}$ to test its effect on extraction beam.

\subsubsection{Optics and Beam Distribution at Compton IP}

Fig. 15 shows the disrupted $\beta$ functions and dispersion in the extraction line for the Nominal parameter option C11, when detector solenoid field is turned on, the initial vertical orbit angle at IP is $y^{\prime}(I P)=50 \mu \mathrm{rad}$, and there is no 
compensation. As one can see, the $\beta$ functions are not significantly affected, but there is an increase of vertical dispersion created by the solenoid and by the vertical orbit angle at IP.

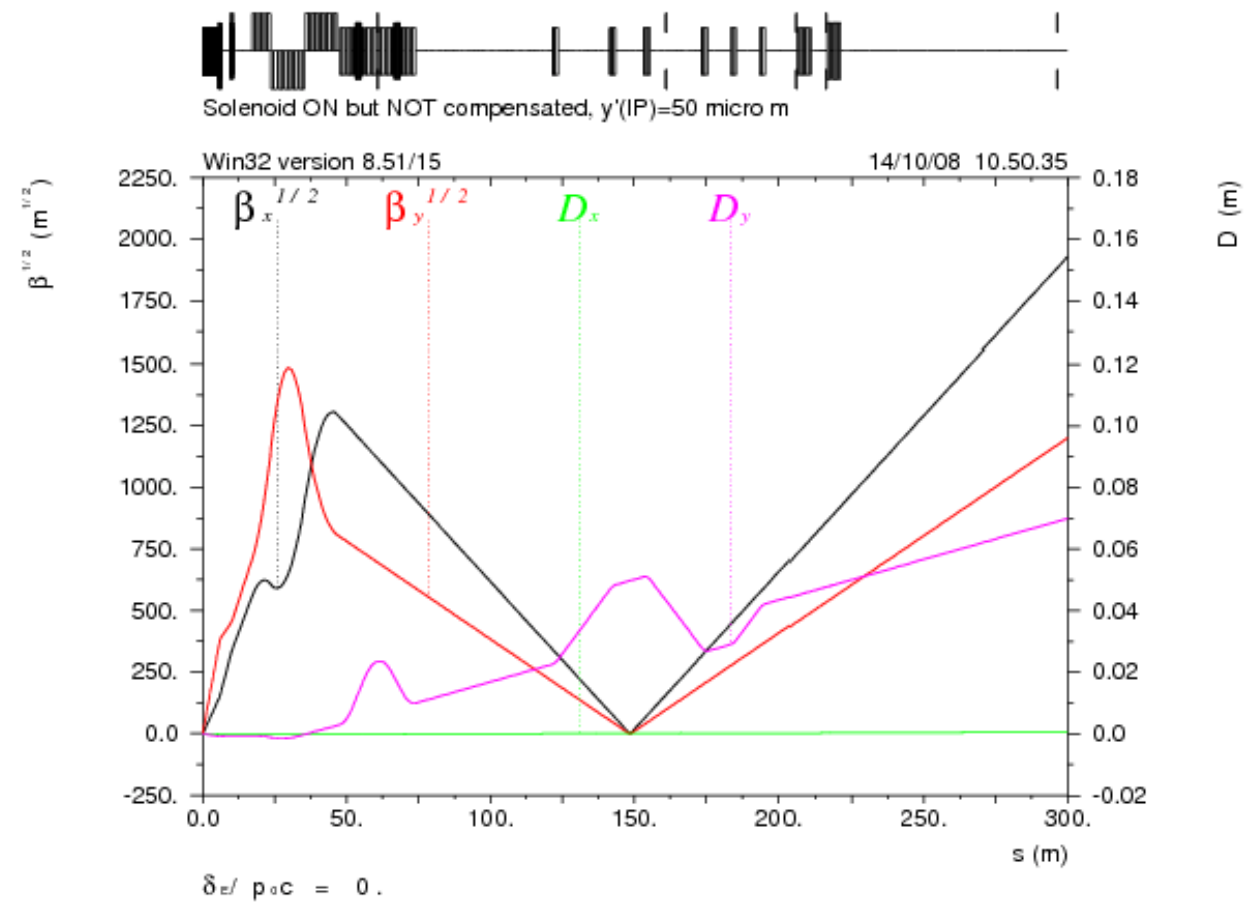

Figure 15: Disrupted $\beta$ functions and dispersion in the $14 \mathrm{mrad}$ extraction line for the Nominal parameter option when solenoid field is turned on, and the IP vertical orbit angle is $y^{\prime}(I P)=50 \mu \mathrm{rad}$.

The corresponding $x, y$ orbits are shown in Fig. 16. If the orbit and dispersion deviations are not compensated, they can lead to larger particle amplitudes and therefore higher beam losses in the extraction line.

For accurate polarization measurements the beam horizontal and vertical orbit angles at IP and Compton IP must be the same, i.e. in this case $y^{\prime}(I P)=y^{\prime}(C I P)=50 \mu \mathrm{rad}$ and $x^{\prime}(I P)=x^{\prime}(C I P)=0$. However, the vertical orbit angle at CIP in this case is $y^{\prime}(C I P)=-14 \mu \mathrm{rad}$, as calculated by MAD in Fig. 16, and therefore must be corrected to satisfy the above requirement. For a local adjustment of vertical orbit, four vertical dipole correctors (labeled VKPOL1, VKPOL2, VKPOL3 and VKPOL4) are placed near the four polarimeter bends symmetrically around the CIP. In Fig. 17 these correctors are set to adjust the CIP vertical angle to $50 \mu \mathrm{rad}$ and also to cancel vertical orbit after the polarimeter chicane. However, the vertical dispersion at CIP is not significantly affected by these correctors, and therefore it remains large as shown in Fig. 15. A similar system of four horizontal dipole correctors can be used to control the horizontal orbit at CIP.

DIMAD tracking of disrupted primary beam with the solenoid field turned on and with the orbit correction at Compton IP, as described above, was performed using full beam files with $7 \cdot 10^{4}$ particles. The results of $x-y$ distributions at CIP are shown in Fig. 18 for the Nominal, Low Y-emittance and Low Power parameter options without and with $y$-offset at IP. Comparison with the unperturbed $x-y$ distributions in Fig. 13 shows a larger beam spread in the direction of negative $y$-values in Fig. 18. As a result, the $\langle x\rangle,\langle y\rangle$ values of the beam core offset are increased by 
40-60 $\mu \mathrm{m}$ compared to Fig. 13. This is caused by the large vertical dispersion and $x$ and $y$ orbits at Compton IP produced by the solenoid.

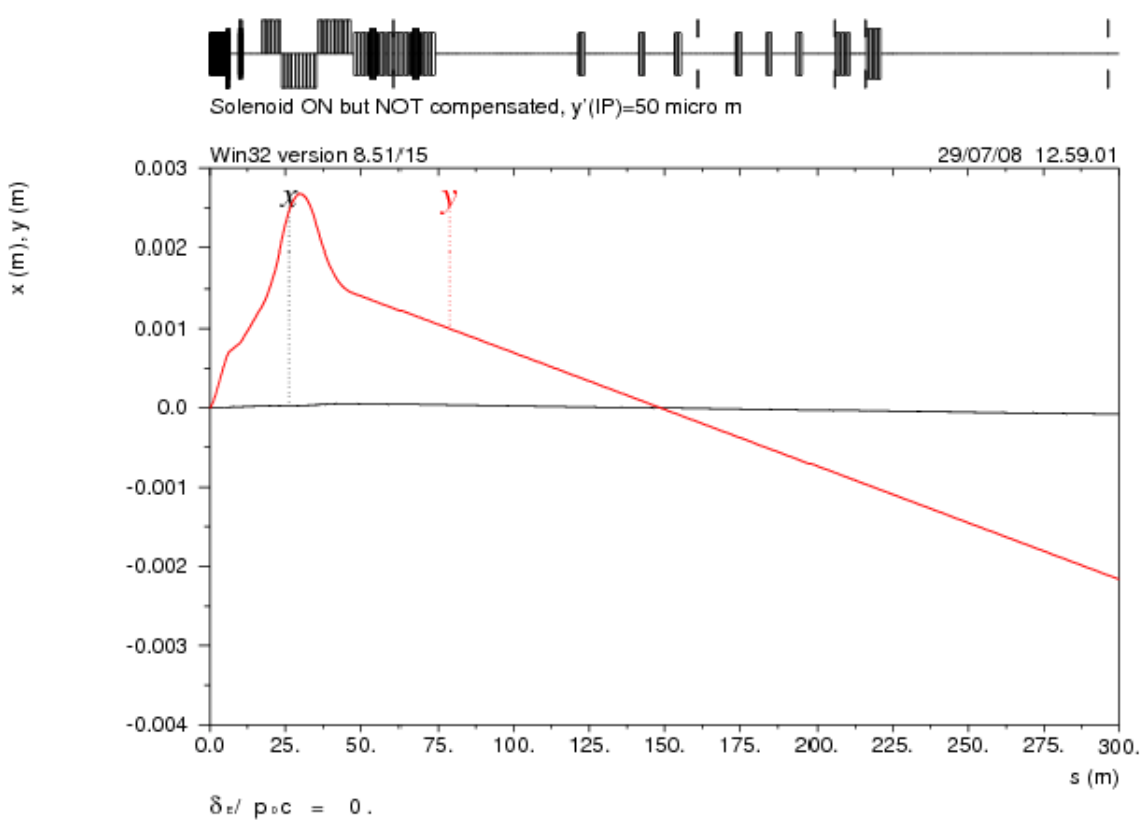

Figure 16: $X$ and $Y$ orbit in the 14 mrad extraction line with the SiD solenoid field turned on, without compensation. Vertical orbit angles at IP and CIP are $y^{\prime}(I P)=50 \mu \mathrm{rad}$ and $y^{\prime}(\mathrm{CIP})=-14 \mu \mathrm{rad}$.

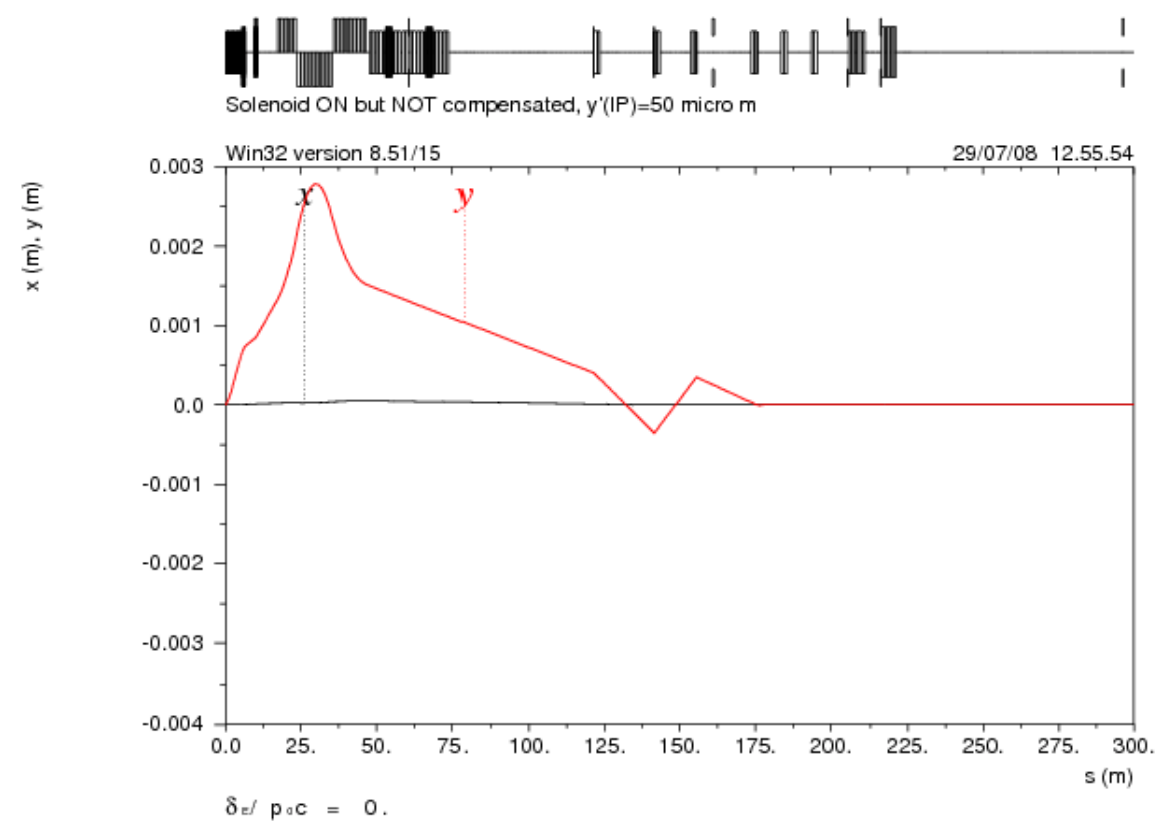

Figure 17: $X$ and $Y$ orbit in the 14 mrad extraction line with the SiD solenoid field turned on. Four dipole correctors in the polarimeter chicane adjust vertical orbit angle at CIP to $y^{\prime}(C I P)=50 \mu$ rad and cancel downstream orbit. 

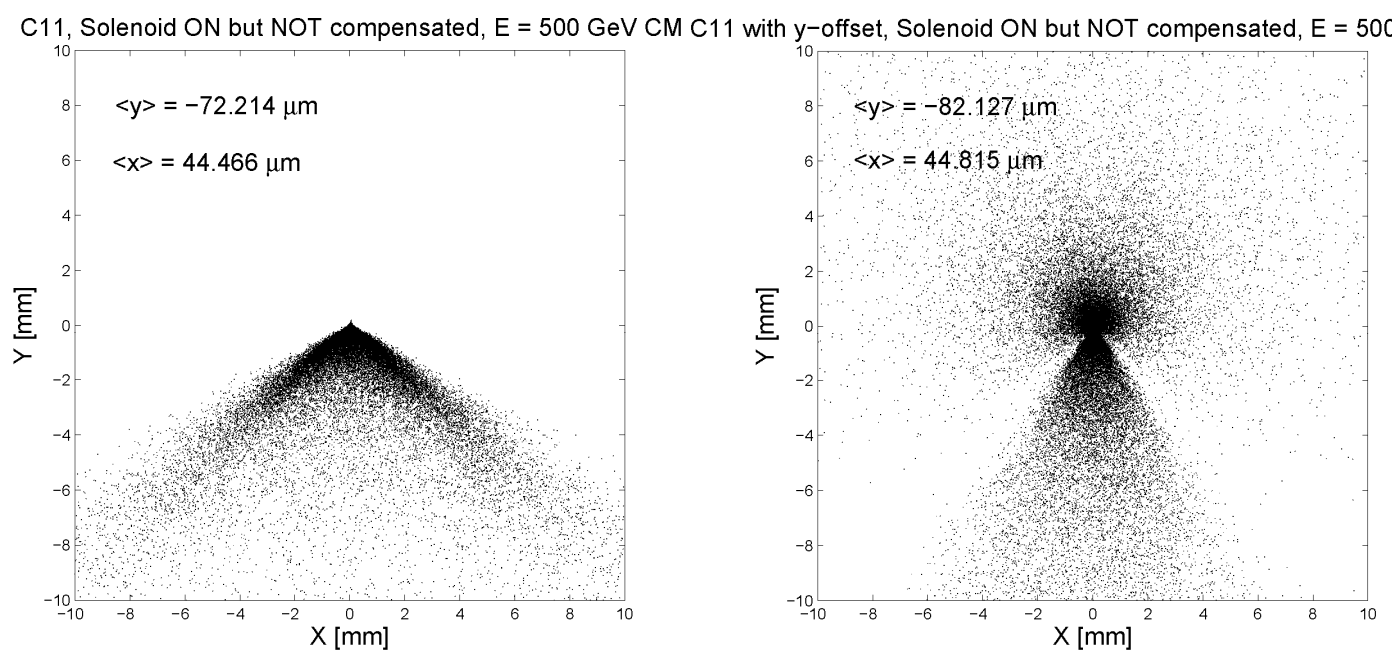

C13, Solenoid ON but NOT compensated, $E=500 \mathrm{GeV}$ CM C13 with y-offset, Solenoid ON but NOT compensated, E = 500 GeV CM
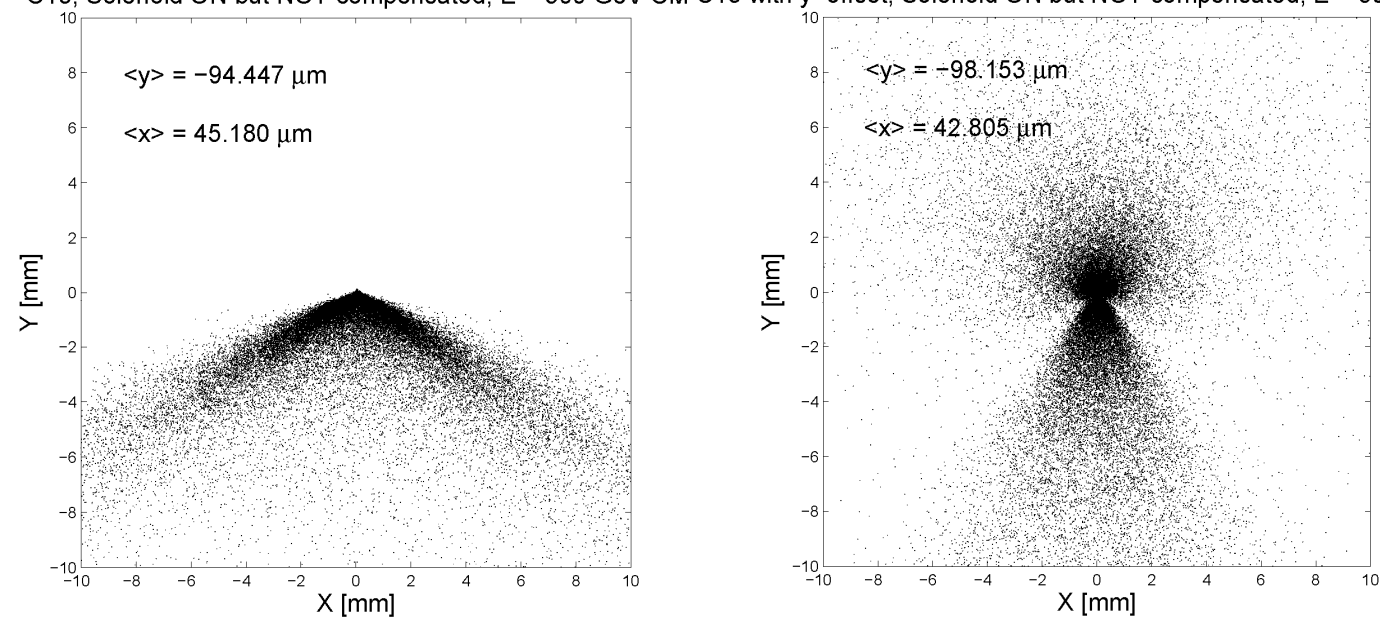

C14, Solenoid ON but NOT compensated, $E=500 \mathrm{GeV} \mathrm{CM} \mathrm{C14} \mathrm{with} \mathrm{y-offset,} \mathrm{Solenoid} \mathrm{ON} \mathrm{but} \mathrm{NOT} \mathrm{compensated,} \mathrm{E} \mathrm{=} \mathrm{500} \mathrm{GeV} \mathrm{CM}$
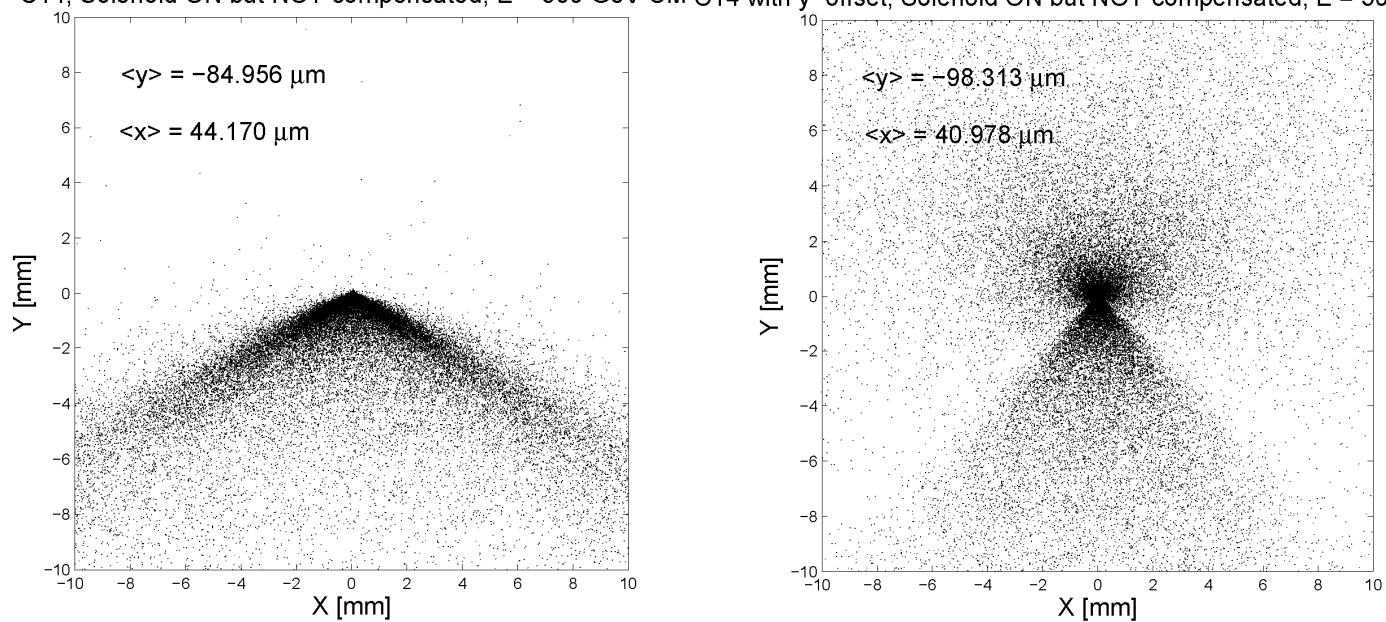

Figure 18: $X-Y$ disrupted beam distributions at Compton IP for the Nominal (top), Large Y-emittance (middle) and Low Power (bottom) parameter options without (left) and with (right) IP $y$-offset, when solenoid field is turned on without compensation. Here $\langle x>$ and $\langle y>$ are the horizontal and vertical offsets of beam core with $|\Delta p / p|<1 \%$. 


\subsubsection{Polarization at Compton IP}

Horizontal field component of the solenoid on beam trajectory in the 14 mrad beam crossing creates vertical beam deflection and may contribute to depolarization in the extraction beam through the Bargmann-Michel-Telegdi (BMT) effect [15]. The BMT spin precession with respect to the electron momentum vector can be written as [16]:

$$
\theta_{\text {spin }}=\gamma \frac{g-2}{2} \theta_{\text {bend }} \equiv \frac{E[G e V]}{0.44065} \theta_{\text {bend }} \text {. }
$$

Depolarization resulting from spin diffusion is given by:

$$
\Delta P=1-\cos \left[\sigma\left(\theta_{\text {spin }}\right)\right],
$$

where $\sigma\left(\theta_{\text {spin }}\right)$ is a standard rms of the spin precession distribution.

Polarization at Compton IP for the case of the detector solenoid turned on and corrected $y^{\prime}(C I P)$ was reconstructed using the tracked disrupted beam distribution and assuming $\pm 100 \mu \mathrm{m}$ of the laser spot size at the CIP. The results are shown in Fig. 19 for the three parameter options, with and without IP $y$-offset. Since the beam vertical angular spread is much smaller than the horizontal one, it does not contribute significantly to the spin diffusion.

The 14 mrad extraction optics is designed to have the transfer matrix term $R_{22}$ from IP to CIP equal to -0.5 , so that polarization at CIP is close to the luminosity weighted polarization at IP. Comparison of Fig. 11 and Fig. 19 shows a good agreement between the luminosity weighted polarization at IP and polarization projection at CIP, especially under ideal conditions without IP offset. This means that the solenoid effect on beam polarization is relatively small. It is expected that polarization measurement accuracy of $0.25 \%$ should be achieved [17].

\section{LOCAL SOLENOID CORRECTION}

The detector solenoid field generates vertical orbit and dispersion due to the $14 \mathrm{mrad}$ crossing angle, as well as a weak focusing and coupling on the extracted beam. The initial vertical orbit angle $y^{\prime}(I P)$ also creates a downstream vertical orbit. These effects perturb the beam conditions at Compton IP and may result in higher beam loss due to larger particle amplitudes. The four correctors described in the previous section can correct the vertical orbit at CIP and downstream of the polarimeter chicane, but they do not affect the upstream orbit and dispersion. For efficient correction of the upstream part of the orbit, one needs dipole correctors placed as close as possible to the solenoid.

\subsection{Local Correction Scheme}

The proposed local correction system includes a special detector integrated dipole coil (DID) $[5,18]$ built on the top of the solenoid and horizontal and vertical dipole corrector windings on each of the two extraction SC quadrupoles QDEX1 and QFEX2A as shown in Fig. 20. Historically, the DID was initially proposed for correction of the solenoid before IP, but later it was realized that it is more beneficial for solenoid compensation after IP in order to minimize the detector background. In the latter case the DID field must be reversed, hence its name was changed to anti-DID. 


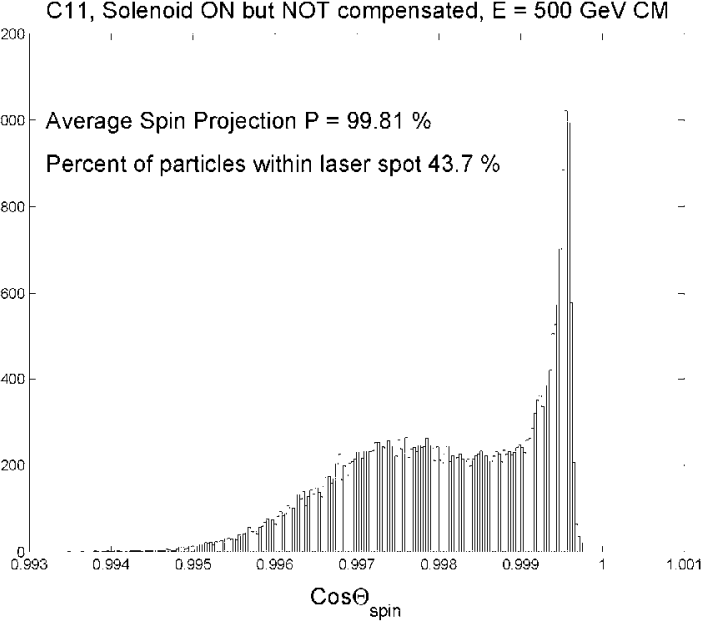

C13, Solenoid ON but NOT compensated, $E=500 \mathrm{GeV} \mathrm{CM}$

Average Spin Projection $\mathrm{P}=99.68 \%$

Percent of particles within laser spot $30.7 \%$

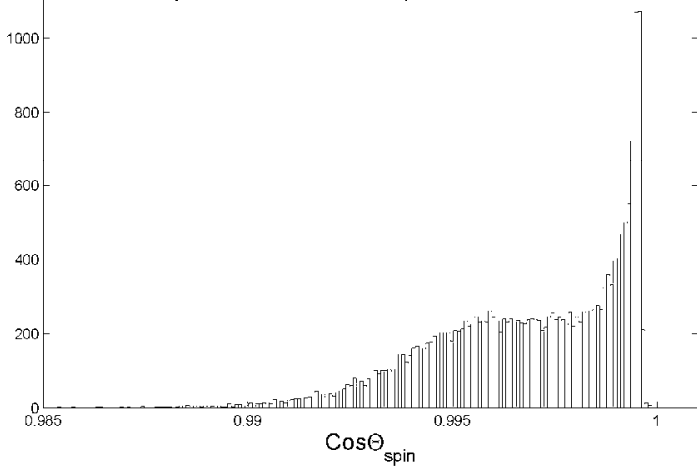

C14, Solenoid ON but NOT compensated, E $=500 \mathrm{GeV} \mathrm{CM}$

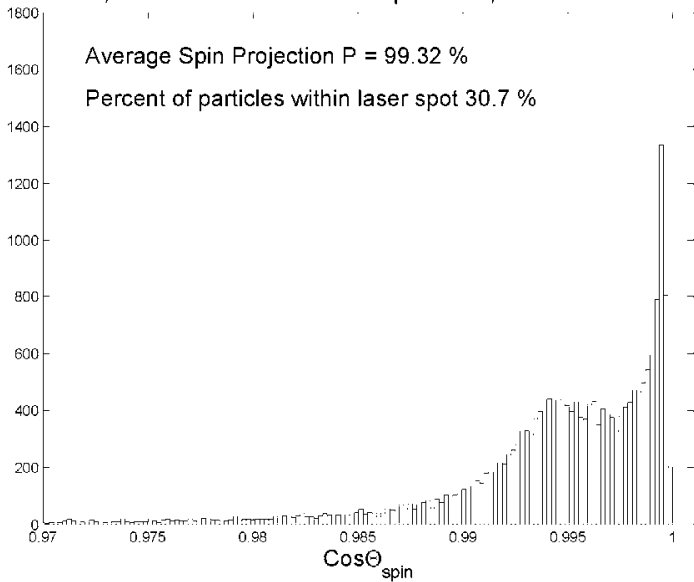

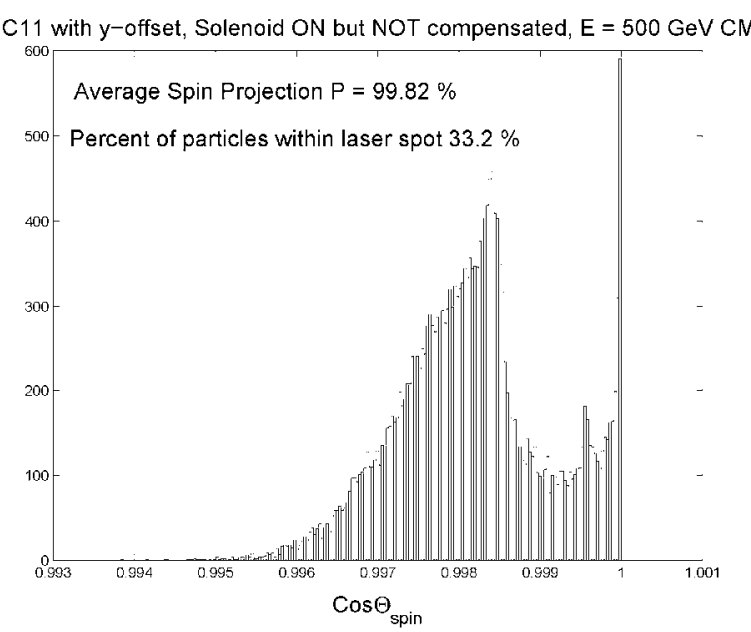

C13 with $y$-offset, Solenoid ON but NOT compensated, $E=500 \mathrm{GeV} \mathrm{CM}$

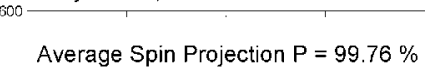

500 - Percent of particles within laser spot $27.3 \%$

400

300

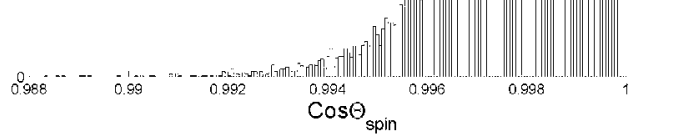

C14 with $y$-offset, Solenoid ON but NOT compensated, $E=500 \mathrm{GeV} \mathrm{CM}$

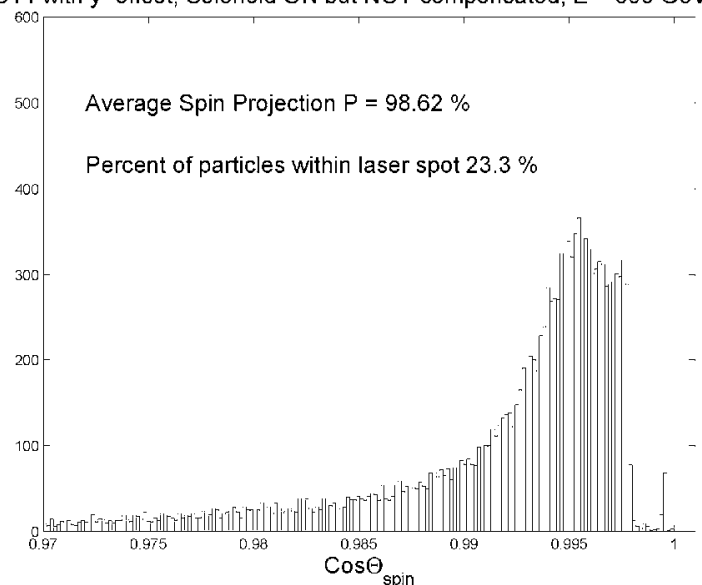

Figure 19: Polarization at the Compton IP for the Nominal (top), Large Y-emittance (middle) and Low Power (bottom) parameter options without (left) and with (right) IP $y$-offset, where the detector solenoid field is turned on, and vertical orbit angle at Compton IP is adjusted to $y^{\prime}(C I P)=50 \mu \mathrm{rad}$. 


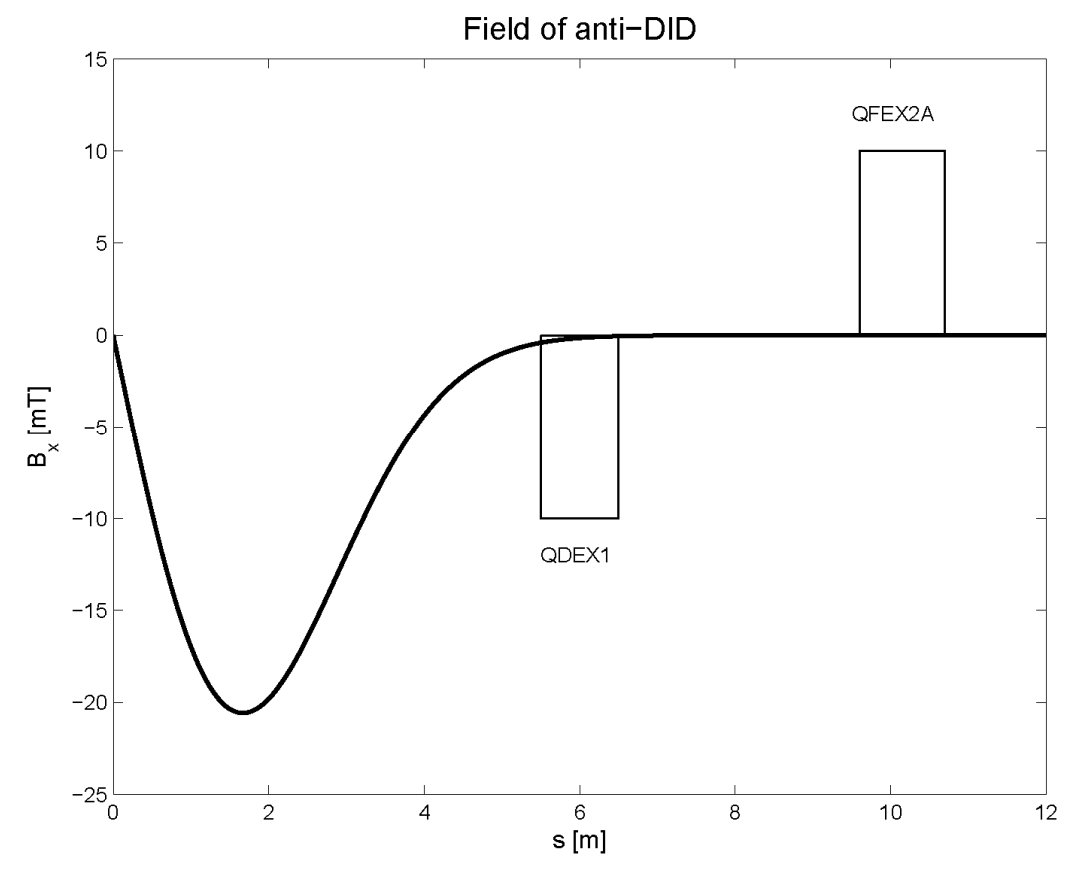

Figure 20: Horizontal field of the anti-DID coil on the detector solenoid starting from IP. Horizontal and vertical dipole corrector windings are included on each of the QDEX1 and QFEX2A quadrupoles.

The dipole corrector windings on the two SC quadrupoles (labeled as VKEX1, VKEX2, HKEX1 and HKEX2) were assumed to have the same length as the quadrupoles, but were modeled as thin correctors in MAD. The field in these correctors can be set up to cancel $x$ and $y$ orbit after QFEX2A, caused both by the solenoid and the initial $y^{\prime}(I P)$. For a given optics and known SiD solenoid and anti-DID fields, the corrector fields can be found by MAD and expressed as a function of the solenoid field, beam energy and the IP vertical angle $y^{\prime}(I P)$ as follows:

$$
\begin{aligned}
& B_{V K E X 1}^{x}[k G]=-0.11087 \cdot F_{S}-13.9312 \cdot y^{\prime}(I P)[\text { mrad }] \cdot F_{E} \\
& B_{V K E X ~}^{x}[k G]=0.23235 \cdot F_{S}+10.9935 \cdot y^{\prime}(I P)[\mathrm{mrad}] \cdot F_{E} \\
& B_{H K E X 1}^{y}[k G]=-0.23068 \cdot F_{S} \cdot y^{\prime}(I P)[\mathrm{mrad}]-0.00651 \cdot \frac{F_{S}^{2}}{F_{E}} \\
& B_{H K E X 2_{2}}^{y}[k G]=0.10409 \cdot F_{S} \cdot y^{\prime}(I P)[\mathrm{mrad}]+0.00289 \cdot \frac{F_{S}^{2}}{F_{E}}
\end{aligned}
$$

where $F_{S}=B_{S} / B_{S 0}$ is the solenoid field scaling factor, $F_{E}=E / E_{0}$ is the beam energy scaling factor, and $B_{S o}$ and $E_{0}=250$ $\mathrm{GeV}$ are the nominal solenoid field and nominal beam energy, respectively. Note that the different analytical dependence for the $B_{x}$ and $B_{y}$ fields in the above formulas is due to the different sources of the $x$ and $y$ orbits. The $y$ orbit is caused by the $x$-angle between the beam and the solenoid as well as by the non-zero IP angle $y^{\prime}$ (IP), while the small $x$-orbit is the result of coupling of vertical orbit into $x$ plane. At much lower beam energy, the $x$-orbit can be significantly larger because of the stronger solenoid coupling. This is reflected in the terms proportional to $1 / F_{E}$ in the above formulas for $B_{y}$ field. 
Effect of the anti-DID field on dispersion and orbit without the dipole correctors was verified in MAD. Fig. 21 shows that the anti-DID alone reduces the CIP dispersion by $\sim 40 \%$ as compared to Fig. 15, and it gives almost a factor of 3 reduction of vertical orbit in the IP region in Fig. 22 as compared to Fig. 16.

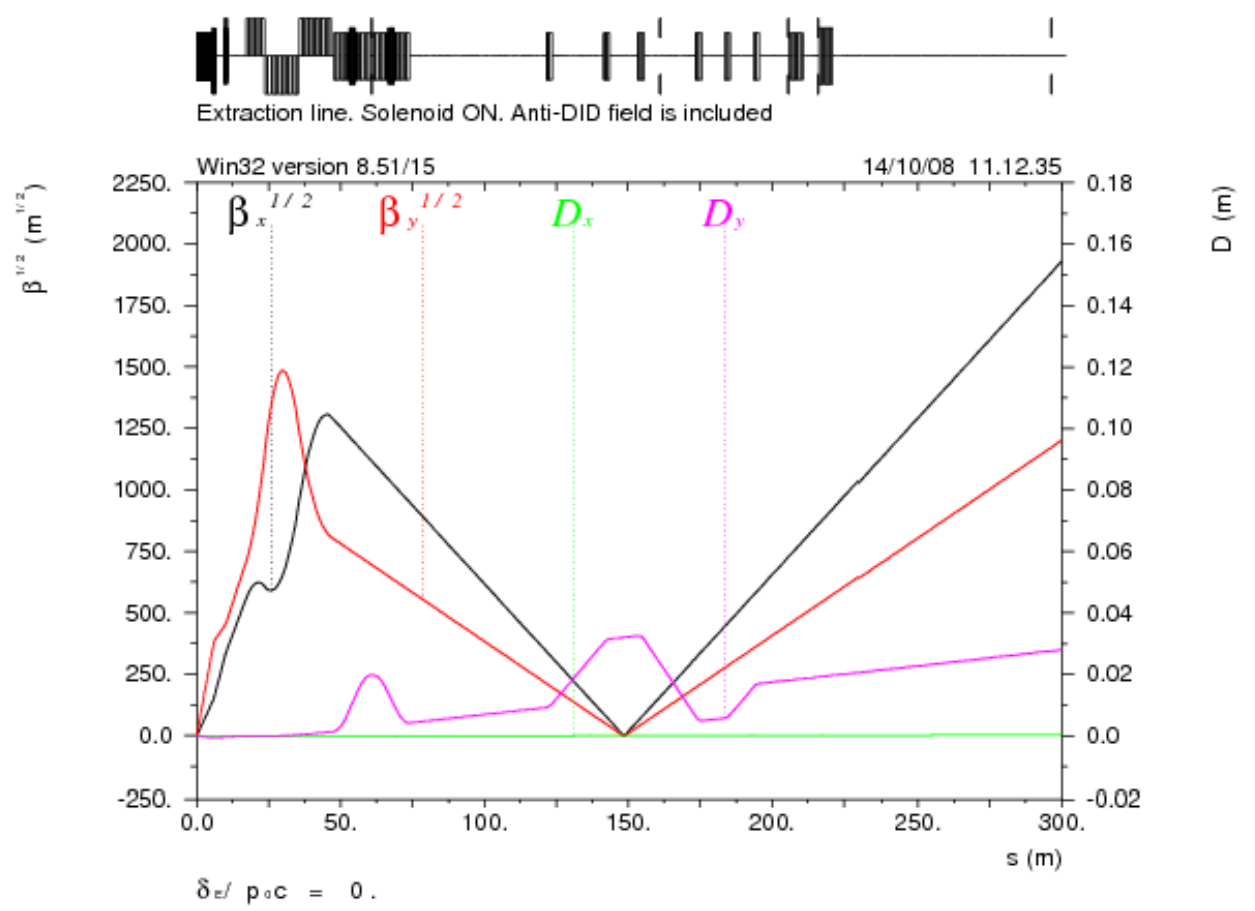

Figure 21: Disrupted $\beta$ functions and dispersion in the $14 \mathrm{mrad}$ extraction line for the Nominal parameter option when the solenoid and anti-DID field are turned on, and the IP vertical orbit angle is $y^{\prime}(I P)=50 \mu$ rad. All dipole correctors are turned off.

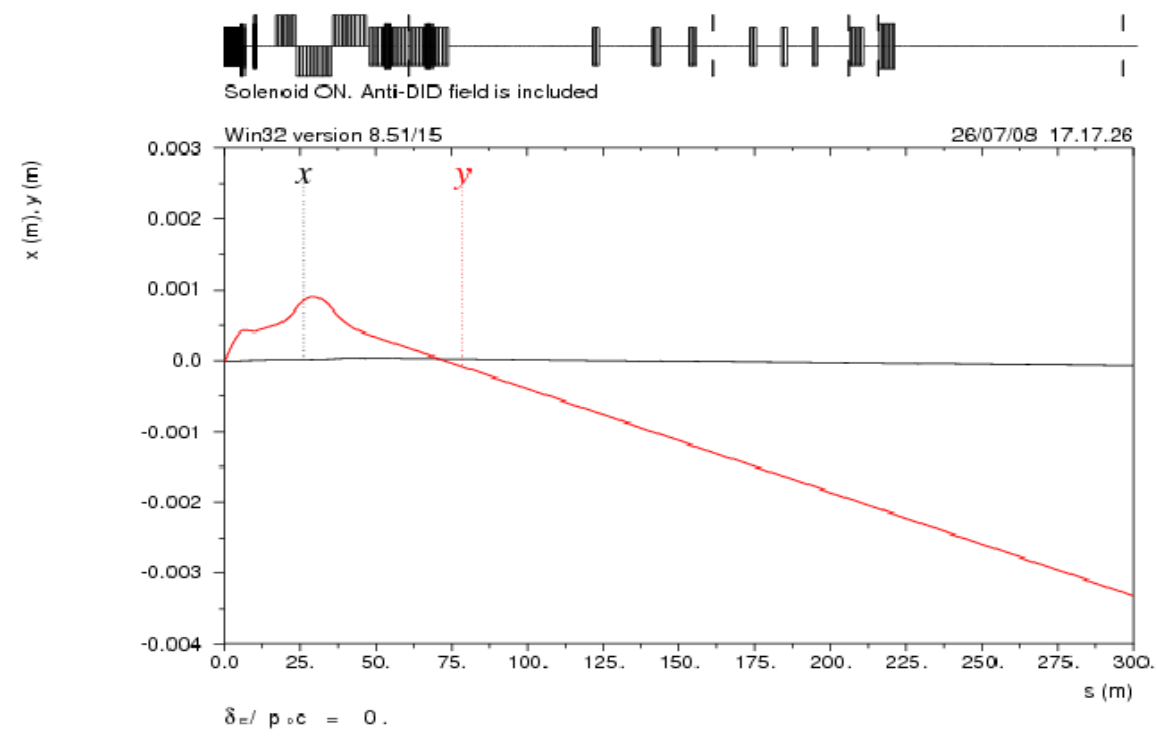

Figure 22: $X$ and $Y$ orbit in the 14 mrad extraction line when the solenoid and anti-DID field are turned on and the IP vertical orbit angle is $y^{\prime}(I P)=50 \mu \mathrm{rad}$. All dipole correctors are turned off. 
The effects of both the anti-DID field and the local correctors are shown in Fig. 23 and 24. The orbit in Fig. 24 is perfectly canceled downstream of the QFEX2A, except near the Compton IP where the local polarimeter correctors are set to make $y^{\prime}(C I P)=50 \mu \mathrm{rad}$. Although the solenoid correctors are not specifically designed to compensate the solenoid dispersion, they provide almost complete correction as shown in Fig. 23, because their positions are close to the solenoid. This compensation should be sufficient for polarization measurement.

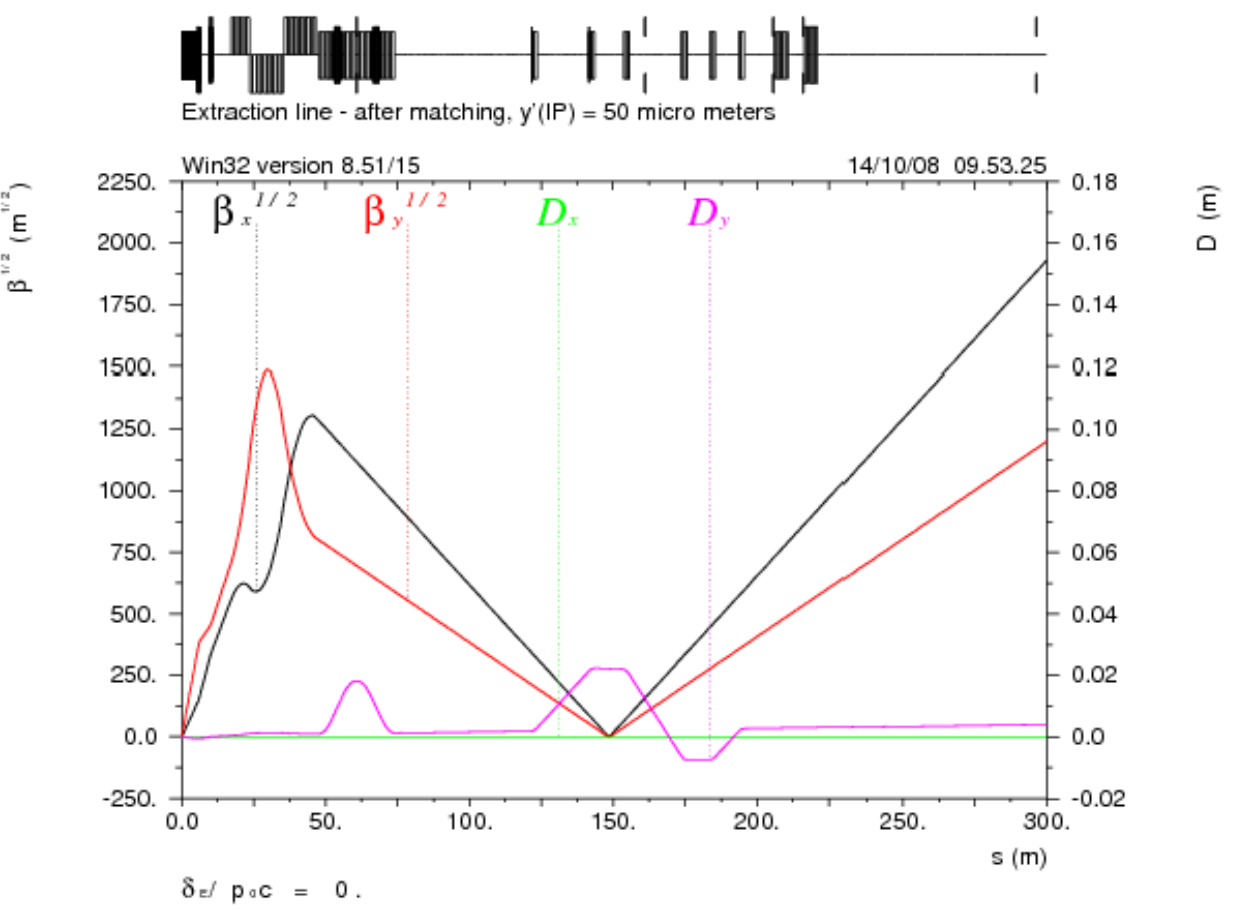

Figure 23: Disrupted $\beta$ functions and dispersion in the $14 \mathrm{mrad}$ extraction line for the Nominal parameter option when the solenoid field, anti-DID field and dipole correctors are turned on. The IP vertical orbit angle is $y^{\prime}(I P)=$ $50 \mu \mathrm{rad}$, and the Compton IP angle is corrected to $y^{\prime}(\mathrm{CIP})=50 \mu \mathrm{rad}$.

The solenoid weak focusing creates a shift of the low beta waist away from Compton IP resulting in a larger beam size at the CIP. This effect can be compensated by using two quadrupole corrector coils on the extraction quadrupoles. These correctors can be set up to maintain the point-to-point focusing from IP to CIP by adjusting the transfer matrix terms $R_{12}$ and $R_{34}$ to zero.

Previous studies did not find a significant effect of solenoid coupling on the extraction beam properties; therefore no coupling correction has been considered. 

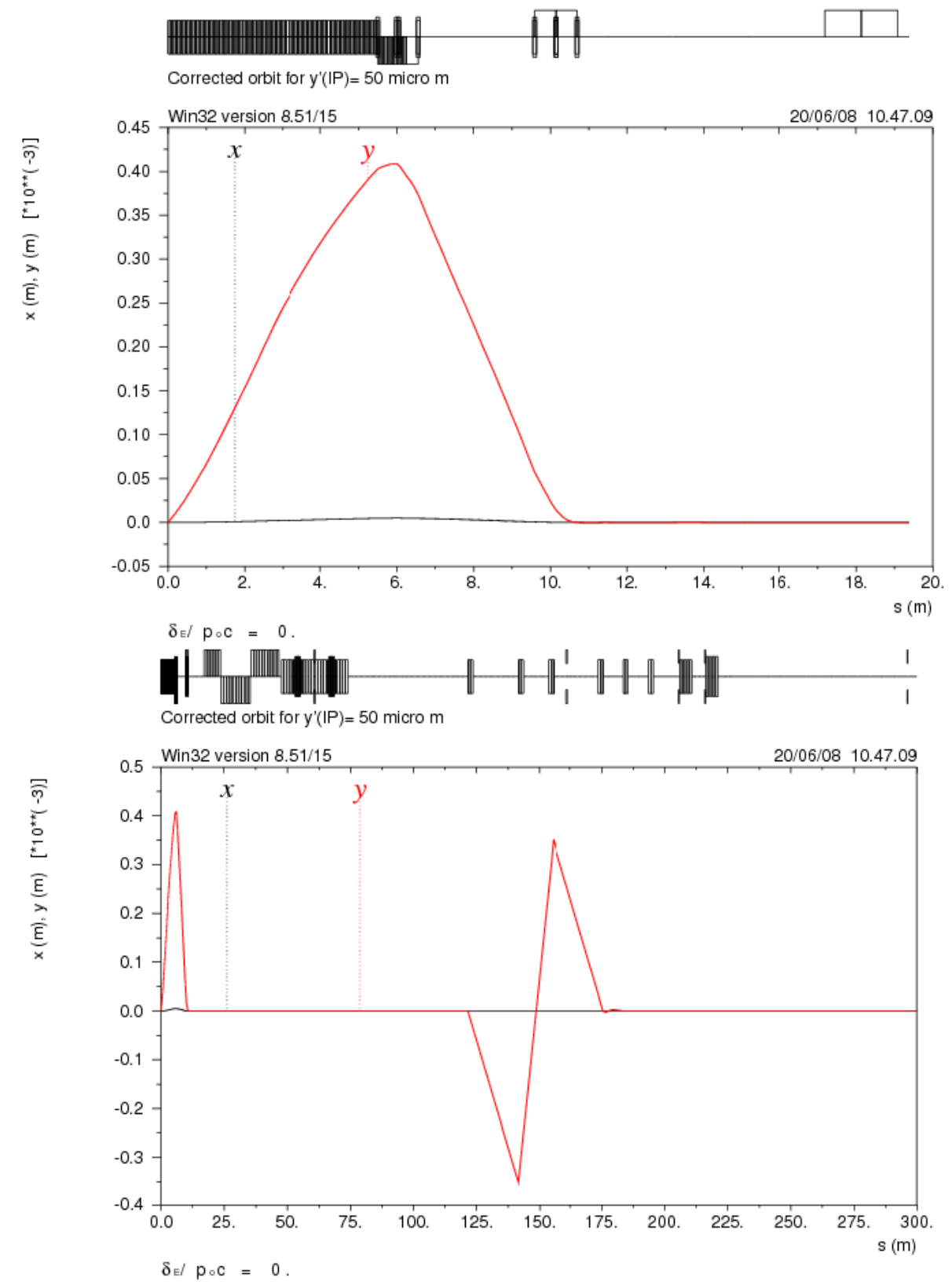

Figure 24: $X$ and $Y$ orbit in the 14 mrad extraction line when the solenoid field, anti-DID field and dipole correctors are turned on. The IP vertical orbit angle is $y^{\prime}(I P)=50 \mu \mathrm{rad}$, and the Compton IP angle is corrected to $y^{\prime}(C I P)=50 \mu \mathrm{rad}$. The upper plot shows local correction in the first $20 \mathrm{~m}$ after IP.

\subsection{Beam Distribution at the Compton IP}

DIMAD tracking of $7 \cdot 10^{4}$ particles was performed to verify the disrupted beam properties at the Compton IP when the SiD solenoid field, anti-DID field and all correctors are turned on. The results of $x-y$ and $x^{\prime}-y^{\prime}$ distributions for the Nominal, Low Y-emittance and Low Power parameters are shown in Fig. 25-29. 

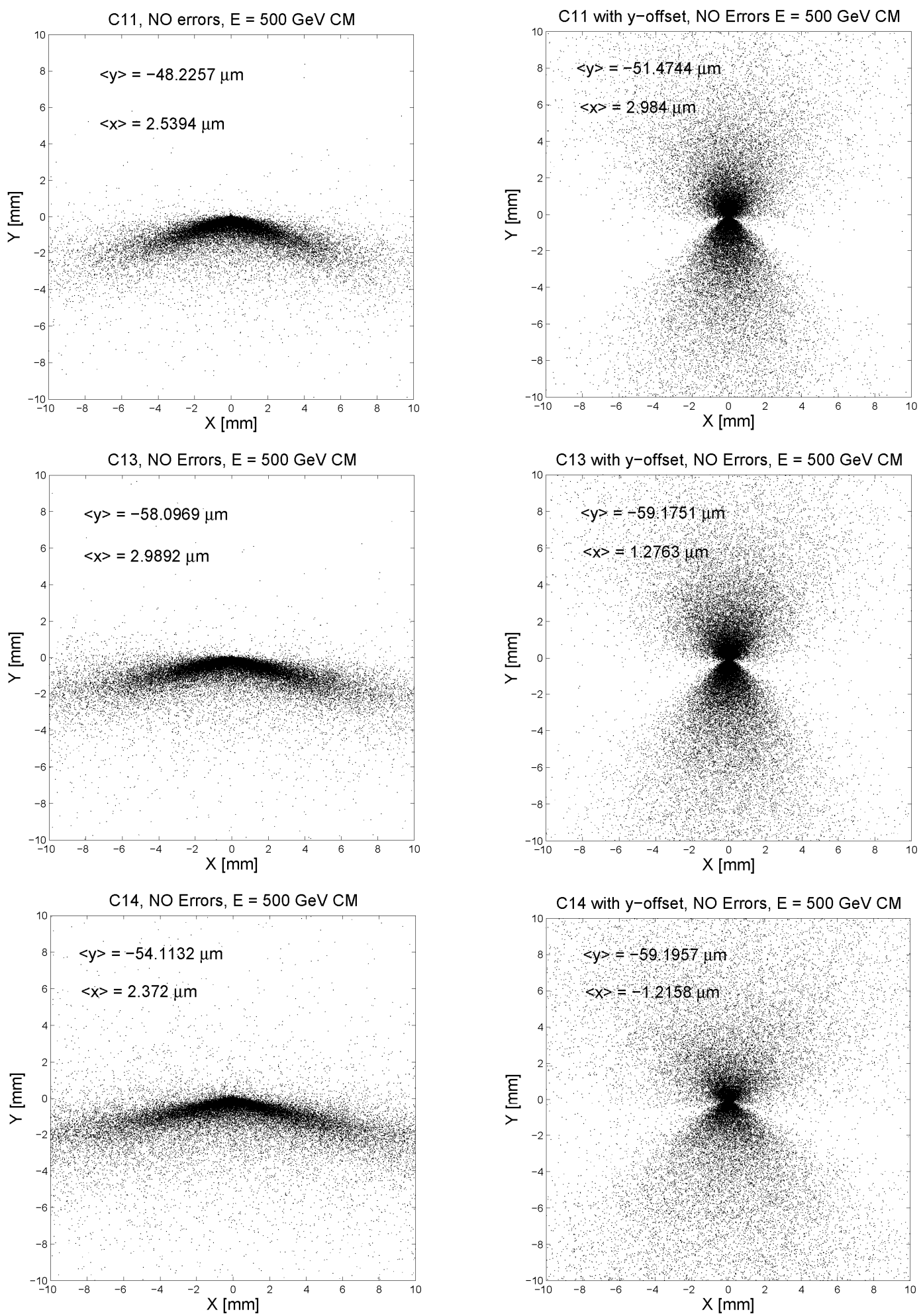

Figure 25: $X-Y$ disrupted beam distributions at Compton IP for the Nominal (top), Large Y-emittance (middle) and Low Power (bottom) parameter options without (left) and with (right) IP $y$-offset, when the solenoid field, anti-DID field and dipole correctors are turned on. Here $<x>$ and $\langle y\rangle$ are the horizontal and vertical offsets of beam core with $|\Delta p / p|<1 \%$. 
Let us remind that the tracking included both colliding beams in one particle file. This increases statistics without changing the distribution in the ideal cases without IP offset. In cases with IP $y$-offset, the two beams have initially large opposite non-zero vertical angles at IP which translate into opposite spread of vertical tails at Compton IP as shown in Fig. 25-29.

Comparison of Fig. 25 and 18 shows that the solenoid compensation removes most of the $\langle x\rangle,\langle y\rangle$ beam core offset and the vertical dispersion spread induced by the solenoid at Compton IP. As a result, the corrected distribution is very close to the distribution without the solenoid. The small difference in $\langle y>$ values between Fig. 25 and 13 is due to the small residual perturbation of vertical dispersion remaining after correction.

Since the vertical orbit at Compton IP is adjusted using the four local correctors to have the same angle as at IP, i.e. $y^{\prime}(C I P)=y^{\prime}(I P)=50 \mu \mathrm{rad}$, the $y^{\prime}$ distributions in Fig. 29 are centered at $50 \mu \mathrm{rad}$.

Summary of the $\langle x\rangle,\langle y\rangle$ offsets of the beam core with $|\Delta p / p|<1 \%$, the average spin projection $P$, and the percentage of particles $k$ within the $\pm 100 \mu \mathrm{m}$ laser spot at Compton IP after the solenoid correction is given in Table 2 for the three beam parameter options (without and with $y$-offset).
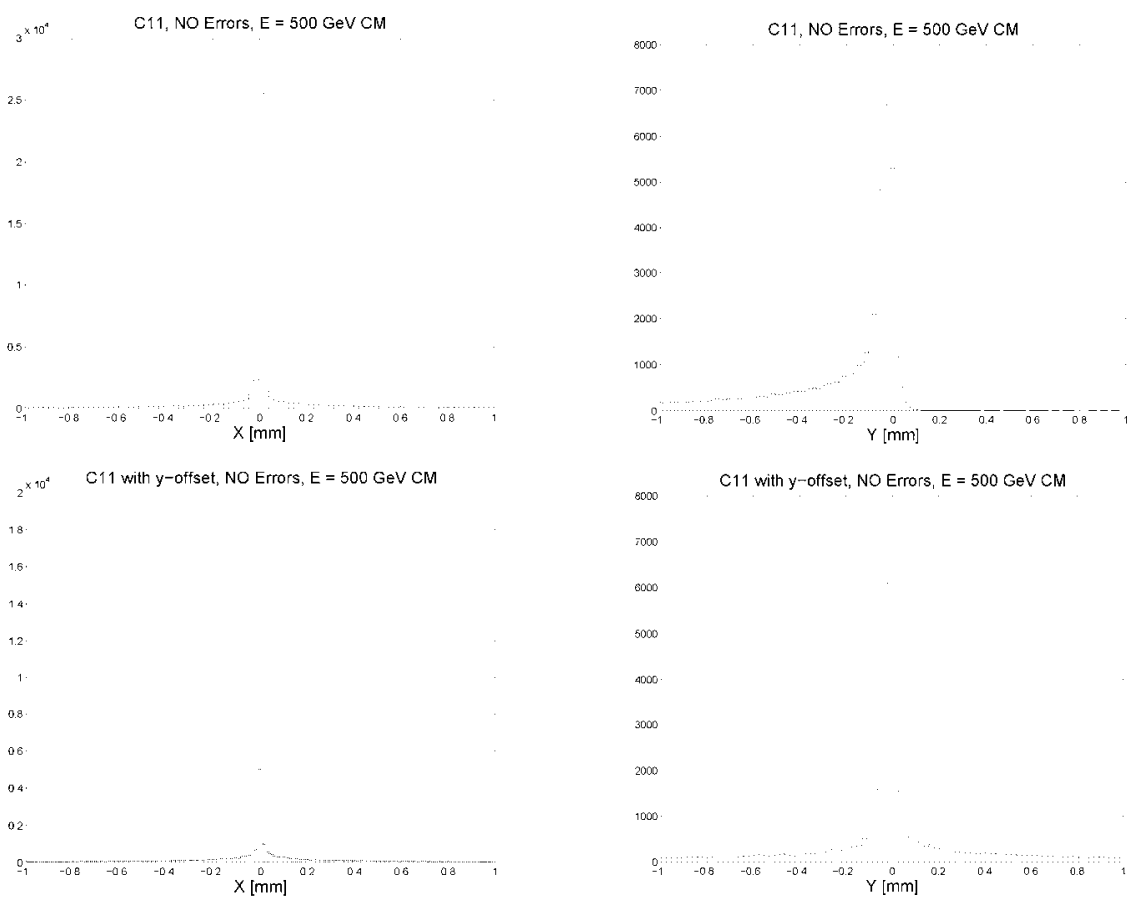

Figure 26: $X$ and $Y$ disrupted beam profiles at Compton IP for the Nominal parameter option without (top) and with (bottom) vertical offset at IP, when the solenoid field, anti-DID field and dipole correctors are turned on. 


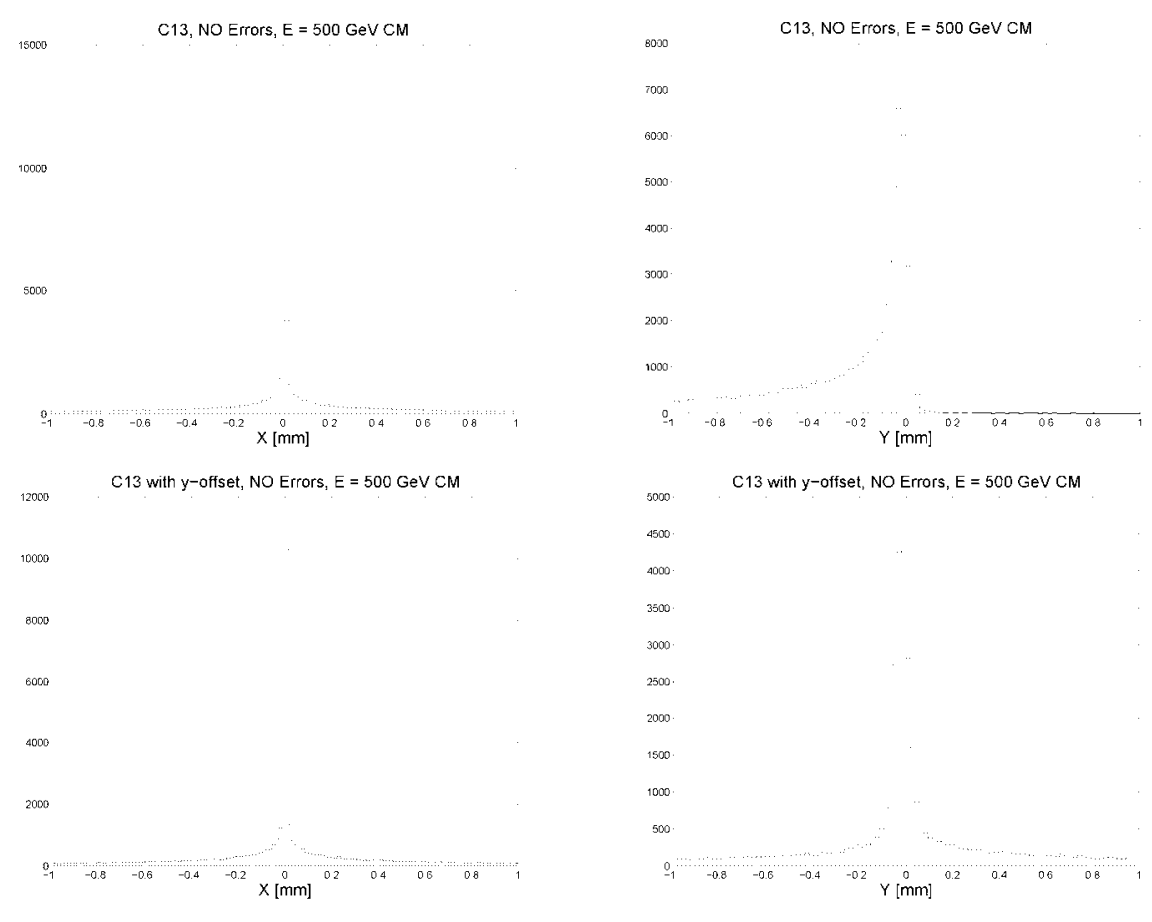

Figure 27: $X$ and $Y$ disrupted beam profiles at Compton IP for the Large $Y$-emittance parameter option without (top) and with (bottom) vertical offset at IP, when the solenoid field, anti-DID field and dipole correctors are turned on.

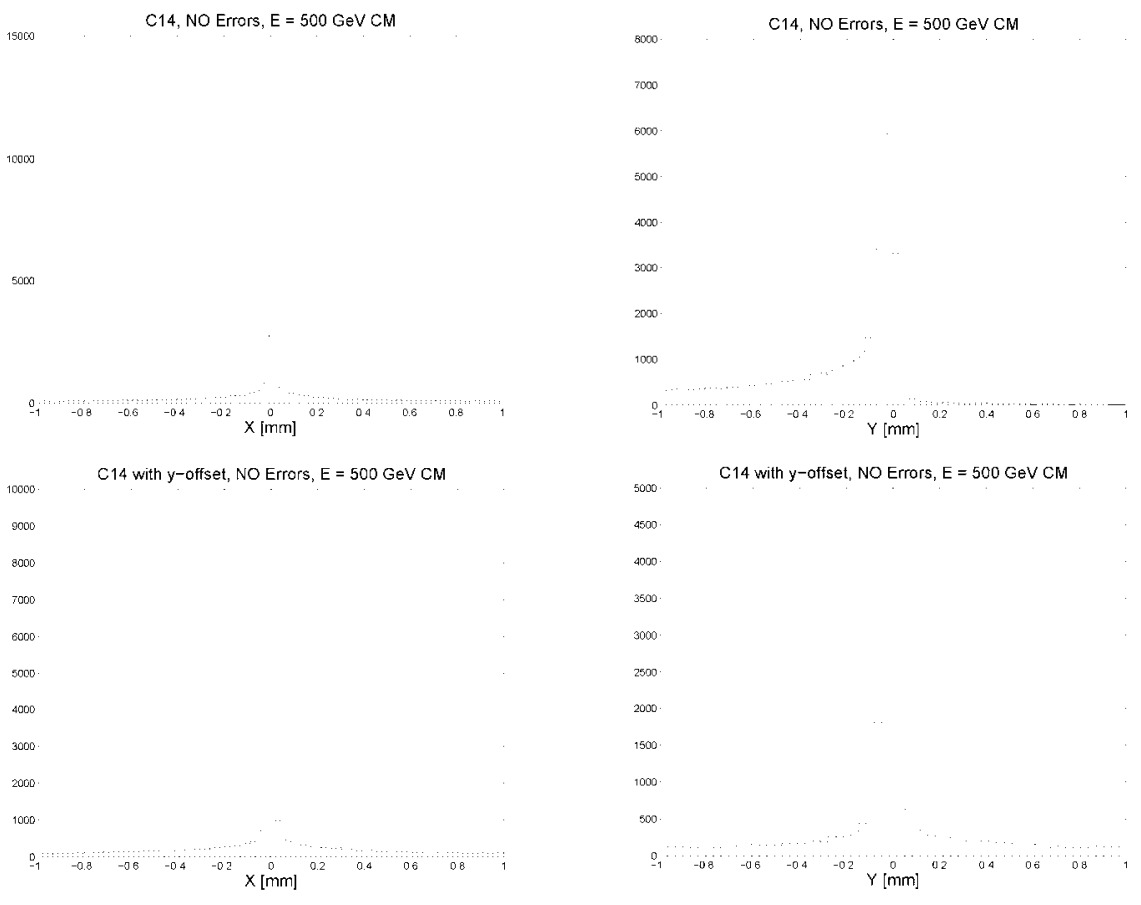

Figure 28: $X$ and $Y$ disrupted beam profiles at Compton IP for the Low Power parameter option without (top) and with (bottom) vertical offset at IP, when the solenoid field, anti-DID field and dipole correctors are turned on. 

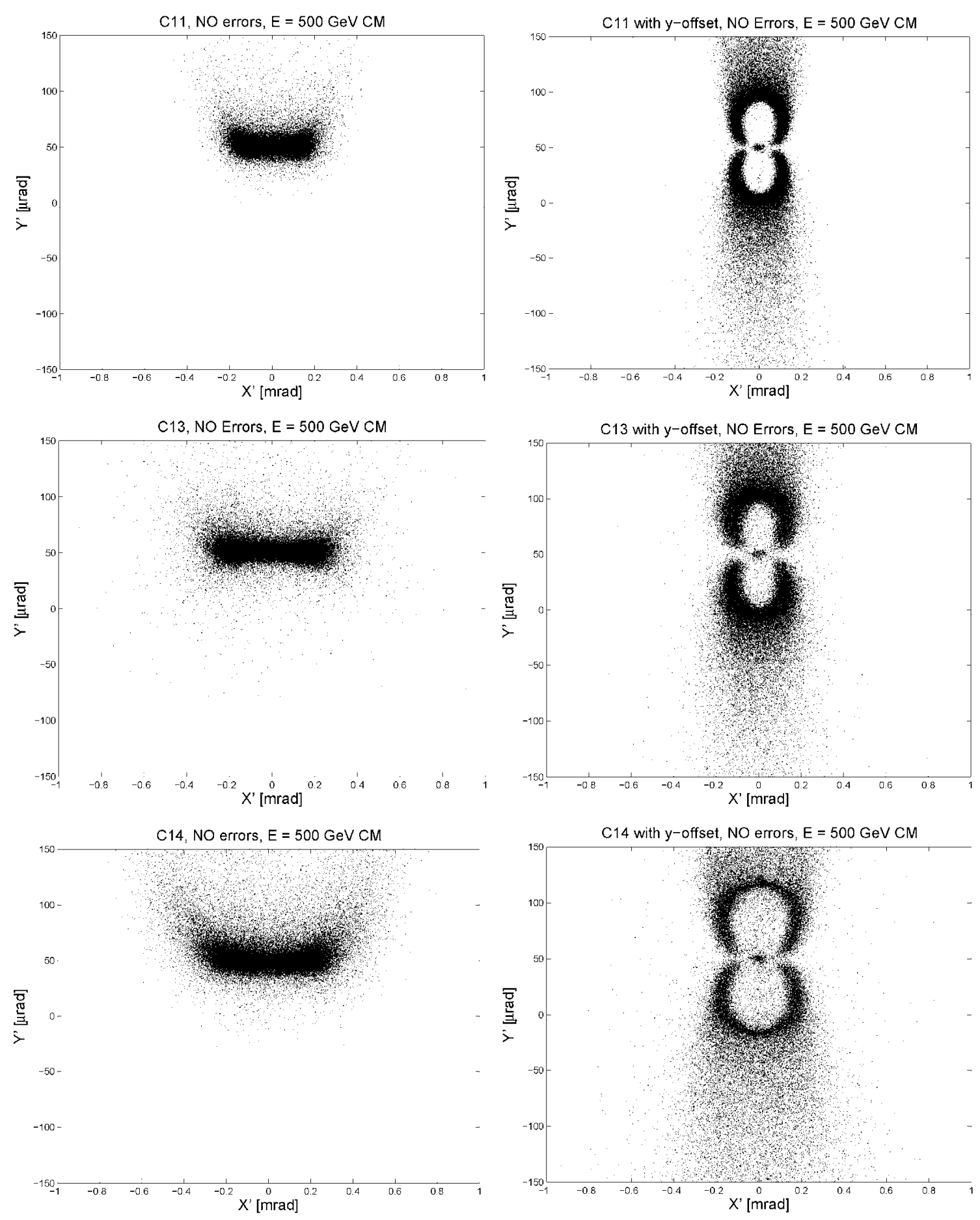

Figure 29: $X^{\prime}-Y^{\prime}$ disrupted beam distributions at Compton IP for the Nominal (top), Large Y-emittance (middle) and Low Power (bottom) parameter options without (left) and with (right) IP $y$-offset, when the solenoid field, anti-DID field and dipole correctors are turned on. 
Table 2: $\langle x>$ and $<y>$ offsets of the beam core with $|\Delta p / p|<1 \%$, the average spin projection $P$, and the percentage of particles $k$ within the $\pm \mathbf{1 0 0} \mu \mathrm{m}$ laser spot at Compton IP for three beam parameter options (without and with $y$-offset) after the solenoid correction.

\begin{tabular}{|l|c|c|c|c|}
\hline Parameter Option & $<\boldsymbol{x}>[\boldsymbol{\mu m}]$ & $<\boldsymbol{y}>[\boldsymbol{\mu m}]$ & $\boldsymbol{P}[\mathbf{\%}]$ & $\boldsymbol{\kappa}$ \\
\hline C11 & 2.5 & -48.2 & 99.82 & 46.3 \\
\hline C11, y-offset & 3.0 & -51.5 & 99.82 & 33.1 \\
\hline C13 & 3.0 & -58.1 & 99.69 & 32.9 \\
\hline C13, y-offset & 1.3 & -59.2 & 99.76 & 27.3 \\
\hline C14 & 2.4 & -54.1 & 99.17 & 34.7 \\
\hline C14, y-offset & -1.2 & -59.2 & 98.34 & 22.8 \\
\hline
\end{tabular}

\section{BEAM POWER LOSS WITH THE SOLENOID AND COMPENSATION}

The primary disrupted beam power losses were calculated using DIMAD tracking. As described earlier, two types of particle data files were used. The relatively low statistics files with $\sim 7 \cdot 10^{4}$ particles for combined beams were used to calculate the relatively large losses on extraction collimators. The high statistics files based on $3.5 \cdot 10^{7}$ particles in the combined beams were used to estimate low losses in the extraction magnets. Since it is known that only particles with the lowest energies and largest IP angles are lost in the magnets, the high statistics files were reduced to much smaller files containing only beam tails where the relative particle energies are below $65 \%$ and/or their IP angles exceed 0.5 mrad. In this tracking, the solenoid, the anti-DID and the compensating dipole correctors are turned on, and the vertical orbit at Compton IP is adjusted to have the same angle as at IP, i.e. $y^{\prime}(C I P)=y^{\prime}(I P)=50 \mu \mathrm{rad}$.

The tracking found no power losses in the extraction quadrupoles for the Nominal and Low Y-emittance parameter options. Therefore only the results for the Low Power option are presented in Table 3. Note that no primary loss was observed in the sensitive SC quadrupoles, and the maximum loss in warm quadrupoles is $1.4 \mathrm{~W}$ for collisions without IP offset and $25 \mathrm{~W}$ for collisions with IP $y$-offset. This loss level is acceptable and should not cause long-term radiation damage. Note that the large beam offsets at IP will be actively corrected in real beam operation in order to maintain high luminosity, therefore the high beam loss caused by the IP offsets will be limited to short periods of time and therefore on average will be significantly lower.

The power losses in the extraction collimators are summarized in Table 4 for the three parameter options without and with IP offset. In this tracking, the fast sweeping kickers were set to sweep the beam on $R=3 \mathrm{~cm}$ circle at dump. Under ideal IP conditions without beam offset, the maximum power loss in a single collimator is near $21 \mathrm{~kW}$. With the large $y$ offset, the maximum collimator loss can be up to $64 \mathrm{~kW}$. However, as mentioned earlier, the IP offsets will be actively corrected and therefore the average loss will be lower. Most of the high loss occurs in the final three collimators COLW1,2,3 which purpose is to clean-up the beam tails before the dump. Since there is no diagnostic in this region, this high loss should be tolerable. The clean-up collimator COLE and protection collimator COLCD are located in the energy and polarimeter diagnostic chicanes where the beam loss is much lower. 
Table 3: Primary disrupted beam loss $(\mathrm{W})$ in the extraction quadrupoles for the Low Power parameter option without and with IP $y$-offset.

\begin{tabular}{|l|c|c|}
\hline Quadrupole & C14 & C14, y-offset \\
\hline QDEX1 (SC) & 0 & 0 \\
\hline QFEX2A (SC) & 0 & 0 \\
\hline QFEX2B & 0 & 0 \\
\hline QFEX2C & 0 & 0 \\
\hline QFEX2D & 0 & 0 \\
\hline QDEX3A & 0 & 0.3 \\
\hline QDEX3B & 0 & 0 \\
\hline QDEX3C & 0.1 & 0 \\
\hline QDEX3D & 0.5 & 0 \\
\hline QDEX3E & 1.0 & 0 \\
\hline QFEX4A & 1.4 & 0.1 \\
\hline QFEX4B & 0 & 0 \\
\hline QFEX4C & 0.4 & 0.4 \\
\hline QFEX4D & 0.3 & 0.3 \\
\hline QFEX4E & 0.1 & 25.4 \\
\hline
\end{tabular}

Table 4: Primary disrupted beam loss (kW) in the extraction collimators for the Nominal, Low Y-emittance and Low Power parameter options without and with IP $y$-offset. Fast sweeping kickers are set to sweep the beam on $R=3 \mathrm{~cm}$ circle at dump.

\begin{tabular}{|l|c|c|c|c|c|}
\hline Parameter Option & COLE & COLCD & COLW1 & COLW2 & COLW3 \\
\hline C11 & 0 & 0 & 0.16 & 0.08 & 0.93 \\
\hline C11, y-offset & 0 & 0.08 & 3.85 & 3.34 & 8.92 \\
\hline C13 & 0 & 0.07 & 1.42 & 1.14 & 5.72 \\
\hline C13, y-offset & 0 & 0 & 2.32 & 1.71 & 8.49 \\
\hline C14 & 0.10 & 0.97 & 10.27 & 8.15 & 21.46 \\
\hline C14, y-offset & 0.34 & 11.53 & 63.66 & 38.60 & 61.34 \\
\hline
\end{tabular}

\section{Acknowledgments}

The authors thank Dr. Deepa Angal-Kalinin and Dr. Robert Appleby for initiating this study and helpful discussions. We are also grateful to Dr. Ken Moffeit for providing graphics in Fig. 2, 3. This work is supported by the U.S. Department of Energy contract DE-AC02-76SF00515. 


\section{References}

[1] J. Brau (Ed.) et al., "ILC Reference Design Report: ILC Global Design Effort and World Wide Study”, FERMILAB-APC (2007).

[2] MAD-8 program, http://mad.web.cern.ch/mad/mad8web/mad8.html .

[3] DIMAD program, http://www-project.slac.stanford.edu/lc/local/AccelPhysics/dimad.pdf .

[4] Y. Nosochkov et al., "14 mrad Extraction Line Optics for Push-Pull”, SLAC-PUB-12856, LCWS-2007-MDI13 (2007).

[5] B. Parker et al., "The Superconducting Magnets of the ILC Beam Delivery System”, PAC07-THPMS091, SLAC-PUB-12832 (2007).

[6] SiD Design Study, http://silicondetector.org/display/SiD/home .

[7] T. Behnke (Ed.) et al., "ILC Reference Design Report Volume 4 - Detectors”, FERMILAB-APC (2007).

[8] K. Moffeit et al., "Proposal to Modify the Polarimeter Chicane in the ILC 14 mrad Extraction Line”, SLACPUB-12425, IPBI-TN-2007-1 (2007).

[9] D. Schulte, "Study of Electromagnetic and Hadronic Background in the Interaction Region of the TESLA Collider”, PhD. Thesis, TESLA-97-08 (1996).

[10] T. Raubenheimer, “Suggested ILC Beam Parameter Range, Rev. 2/28/05”, http://wwwproject.slac.stanford.edu/ilc/acceldev/beampar/Suggested\%20ILC\%20Beam\%20Parameter\%20Space.pdf (2005).

[11] A. Seryi, http://www.slac.stanford.edu/ seryi/ILC_new_gp_files/ (2005).

[12] Y. Nosochkov, T.O. Raubenheimer, K.A. Thompson, "NLC beam properties and extraction line performance with beam offset at IP”, SLAC-PUB-8872, PAC-2001-FPAH065 (2001).

[13] K. Moffeit et al., “Comparison of 2 mrad and 14/20 mrad Crossing Angle Extraction Lines”, IPBI TN-2006-1 (2006).

[14] K. Moffeit et al., IPBI TN-2006-4 (2006).

[15] V. Bargmann, L. Michel, V.L. Telegdi, "Precession of the polarization of particles moving in a homogeneous electro-magnetic field”, Phys. Rev. Lett. 2(10):435-436 (1959).

[16] M. Woods et al., "Luminosity, energy and polarization studies for the linear collider: Comparing e+ e- and e-efor NLC and TESLA”, SLAC-PUB-10353, IPBI-TN-2004-1 (2004).

[17] P.C. Rowson, M. Woods, "Experimental issues for precision electroweak physics at a high luminosity Z factory”, SLAC-PUB-8745 (2000).

[18] A. Seryi et al., "IR Optimization, DID and anti-DID”, SLAC-PUB-11662 (2006). 